DRAFT VERSION NOVEMBER 21, 2014

Preprint typeset using LTEX style emulateapj v. 08/22/09

\title{
THE COS-DWARFS SURVEY: THE CARBON RESERVOIR AROUND SUB-L* GALAXIES ${ }^{1}$
}

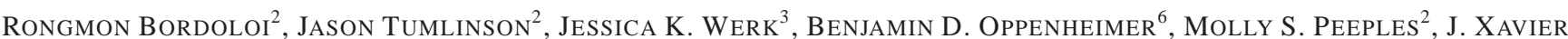 \\ Prochaska ${ }^{3}$, Todd M. Tripp ${ }^{4}$, NeAl Katz ${ }^{4}$, Romeel DaVé ${ }^{9,10,11}$, Andrew J. FoX ${ }^{2}$, Christopher Thom ${ }^{2}$, Amanda Brady \\ FORD $^{5}$, DAVid H. WeinberG ${ }^{7}$, Joseph N. BurChetT ${ }^{4} \&$ JUnA A. KOllmeier $^{8}$ \\ Draft version November 21, 2014
}

\begin{abstract}
We report new observations of circumgalactic gas from the COS-Dwarfs survey, a systematic investigation of the gaseous halos around 43 low-mass $z \leq 0.1$ galaxies using background QSOs observed with the Cosmic Origins Spectrograph. From the projected $1 \mathrm{D}$ and $2 \mathrm{D}$ distribution of $\mathrm{C}$ IV absorption, we find that $\mathrm{C}$ IV is detected out to $\approx 100 \mathrm{kpc}$ (corresponding roughly to $\approx 0.5 \mathrm{R}_{\text {vir }}$ ) of the host galaxies. The $\mathrm{C}$ IV absorption strength falls off radially as a power law and beyond $\approx 0.5 \mathrm{R}_{\mathrm{vir}}$, no $\mathrm{C}$ IV absorption is detected above our sensitivity limit of $\approx 50-100 \mathrm{~m} \AA$. We find a tentative correlation between detected $\mathrm{C}$ IV absorption strength and star formation, paralleling the strong correlation seen in highly ionized oxygen for $\mathrm{L} \sim L^{*}$ galaxies by the COS-Halos survey. The data imply a large carbon reservoir in the CGM of these galaxies, corresponding to a minimum carbon mass of $\gtrsim 1.2 \times 10^{6} \mathrm{M}_{\odot}$ out to $\sim 110 \mathrm{kpc}$. This mass is comparable to the carbon mass in the ISM and exceeds the carbon mass currently in the stars of these galaxies. The $\mathrm{C}$ IV absorption seen around these sub-L* ${ }^{*}$ galaxies can account for almost two-thirds of all $W_{r} \geq 100 \mathrm{~m} \AA \mathrm{C}$ IV absorption detected at low z. Comparing the C IV covering fraction with hydrodynamical simulations, we find that an energy-driven wind model is consistent with the observations whereas a wind model of constant velocity fails to reproduce the CGM or the galaxy properties.

Subject headings: galaxies: evolution,halos_ general—galaxies: quasars: absorption lines_ intergalactic medium
\end{abstract}

\section{INTRODUCTION}

The distribution and physical conditions of gas around galaxies are important ingredients for understanding how galaxies evolve over cosmic time. Acquiring this knowledge entails studying how the galaxies obtain, process, expel and recycle gas from their surroundings. Understanding these processes is further complicated by the indirect nature of the available observations: stars and gas within the galaxies can be accessed and studied directly, but the gas that is being accreted or being expelled is usually either too diffuse or too hot to be observed directly in emission. Hence we typically have to rely on modeling the outcome (stellar mass, color, morphology) of gas processes rather than examining the phenomena themselves. One of the few ways to advance our understanding of gas processes is to observe the circumgalactic medium (CGM) and to correlate the gas properties with the

\footnotetext{
Electronic address: bordoloi@stsci.edu

${ }^{1}$ Based on observations made with the NASA/ESA Hubble Space Telescope, obtained at the Space Telescope Science Institute, which is operated by the Association of Universities for Research in Astronomy, Inc., under NASA contract NAS 5-26555. These observations are associated with program GO12248.

${ }^{2}$ Space Telescope Science Institute, Baltimore, MD

${ }^{3}$ UCO/Lick Observatory, University of California, Santa Cruz, CA

${ }^{4}$ Department of Astronomy, University of Massachusetts, Amherst, MA

${ }^{5}$ Steward Observatory, University of Arizona, Tucson, AZ

${ }^{6}$ Center for Astrophysics and Space Astronomy, Department of Astrophysical and Planetary Sciences, University of Colorado, 389 UCB, Boulder, CO 80309, USA

${ }^{7}$ Department of Astronomy, The Ohio State University, Columbus, $\mathrm{OH}$

${ }^{8}$ Observatories of the Carnegie Institution of Washington, Pasadena, CA

9 University of the Western Cape, Bellville, Cape Town 7535, South Africa

${ }^{10}$ South African Astronomical Observatories, Observatory, Cape Town 7925, South Africa

11 African Institute for Mathematical Sciences, Muizenberg, Cape Town 7945, South Africa
}

host galaxies themselves.

Absorption-line systems observed directly in the spectra of background sources provide one of the few effective tracers of the otherwise invisible gas around galaxies. With this technique it is possible to study the distribution and physical conditions of gas around galaxies. The advent of large galaxy surveys (York et al. 2000; Lilly et al. 2009), and the installation of the Cosmic Origins Spectrograph (COS, Green et al. 2012), aboard the Hubble Space Telescope (HST) have made it possible to systematically map the CGM and to correlate its properties with the host galaxy properties and the large scale environment (Tumlinson et al. 2013; Stocke et al. 2013; Bordoloi et al. 2011; Chen et al. 2010; Prochaska et al. 2011a; Zhu \& Ménard 2013; Werk et al. 2013; Stocke et al. 2014).

The recent "COS-Halos" survey (Tumlinson et al. 2013), mapped the multiphase CGM around $L^{*}$ galaxies at $z=0.15$ - 0.35. This survey utilized the COS spectrograph to acquire UV spectroscopy of background quasars near $44 \mathrm{~L} \gtrsim$ $L^{*}$ galaxies within a $160 \mathrm{kpc}$ impact parameter Werk et al. 2012). The COS-Halos survey was optimized to map the multiphase CGM using O VI and other metal line diagnostics (Tumlinson et al. 2011b; Werk et al. 2013). The COS-Halos program finds that $L^{*}$ star-forming galaxies are surrounded by total CGM gas masses that are comparable to and possibly in excess of their stellar masses (Werk et al. 2014), and that the metal mass in these halos, is comparable to what has been retained in their interstellar gas and dust (Peeples et al. 2014). COS-Halos also finds that passive galaxies are relatively deficient in O VI relative to their star-forming counterparts (Tumlinson et al. 2011b), while the discrepancy in H I is smaller (Thom et al. 2012) and passive galaxies may retain a significant budget of cool CGM gas.

A metals census in the CGM of less luminous galaxies must 
be carried out at lower redshift to exploit the existing largescale, flux-limited galaxy redshift surveys such as the Sloan Digital Sky Survey (SDSS, Abazaiian et al. 2009). However, at $z<0.1$, the OVI doublet at 1031.93 and $1037.62 \AA$ is not sufficiently redshifted to be efficiently observed with the high resolution COS FUV gratings, which have their best sensitivities at wavelengths greater than $1140 \AA$. Thus a census of metals at $<L^{*}$ must use a tracer that is accessible at $z<0.1$ within the COS band.

This paper describes the first results of a new survey ("COS-Dwarfs") of the CGM gas surrounding a sample of $\mathrm{L} \sim 0.006-0.18 L^{*}$ galaxies in the low-redshift Universe using COS. This is a direct follow up of the COS-Halos survey in that we focus on the CGM around low-mass galaxies. Our focus on sub-L*galaxies is primarily motivated by a desire to extend the mass range of the existing surveys to lower masses and thereby cover a total of $\sim 3$ decades in stellar mass (roughly $M_{*} \approx 10^{8-11} \mathrm{M}_{\odot}$ ), to better understand the role of the CGM in galaxy formation.

In this paper we focus on the C IV $\lambda \lambda 1548,1550$ doublet transition, the most accessible tracer of hot and/or highly ionized gas at redshift $z<0.1$. Strong C IV absorption has been used to trace missing metals in the IGM from $z=4$ to today (Steidel 1990; Danforth \& Shull 2008; D'Odorico et al.2010; Simcoe et al. 2011; Cooksey et al. 2010, 2013). This transition has been used extensively at higher redshifts because it is a strong resonant doublet transition giving it a distinct, easily identifiable characteristic. Also, it is observable redward of the Ly $\alpha$ forest, where it becomes easier to identify as it redshifts into the optical passbands at $z>1.5$. C IV absorption line studies have found that the C IV mass density relative to the critical density $\left(\Omega_{\mathrm{CIV}}\right)$ increases smoothly from $z=4$ $\rightarrow 1.5$ (D'Odorico et al. 2010) and maps well onto the $z<1$ measurements (Cooksey et al. 2010). $\Omega_{\text {CIV }}$ increases by a factor of 5 over $z=4 \rightarrow 0$, and the comoving C IV line density $d N_{C I V} / d X$ increases 10-fold over $z \sim 6 \rightarrow 0$ for $W_{r} \geq 0.6 \AA$ (Tilton et al. 2012, Cooksey et al. 2013, Danforth et al. 2014, Burchett et al., in preparation). This may indicate that the CGM around galaxies is being enriched steadily over the last 12 Gyr lifespan of the Universe.

However, at high redshifts it is hard to study the individual host galaxies associated with the C IV absorbers. To study the CGM traced by C IV around galaxies with well defined properties, in a statistical manner, we rely on HST UV spectroscopy of background quasars, which opens the window of the low-z Universe. The pioneering study of Chen et al. (2001) compiled a C IV -galaxy pair sample of 50 galaxies within $200 \mathrm{kpc}$, using HST UV spectroscopy of UV bright quasars at $z \approx 0.4$. They detected C IV absorption within 100 $\mathrm{kpc}$ of their galaxies and found no correlation between absorbers and galaxy morphology or surface brightness. This study was a "blind survey", i.e. it entails taking the spectra of a quasar, finding the absorption line systems first and then looking for associated galaxies in follow up spectroscopic surveys. On the other hand, Borthakur et al. (2013) found, using their small number of absorbers of star-bursting galaxies, that $\mathrm{C}$ IV absorption can be seen even at high impact parameters $(150 \leq \mathrm{R} \leq 200 \mathrm{kpc})$. Individual C IV systems have also been reported, for which either no $\mathrm{L} \gtrsim 0.04 L^{*}$ galaxies were found within $250 \mathrm{kpc}$ (Tripp et al. 2006) or only a very faint dwarf galaxy was detected at $\sim 200 \mathrm{kpc}$ (Burchett et al.|2013).

At higher redshifts, Adelberger et al. (2003, 2005) observed that almost all of their $N_{C I V} \geq 10^{14} \mathrm{~cm}^{-2}$ absorbers are found within $\approx 80 \mathrm{kpc}$ of Lyman-break galaxies (LBGs) at $2 \leq z \leq$ 3. Their findings were based on individual strong absorberLBG pairs, analysis of stacked spectra of close background galaxies and LBG-C IV cross-correlation and LBG autocorrelation functions, suggesting that $\mathrm{C}$ IV have the same spatial distribution as the galaxies. Steidel et al. (2010) examined the mean $\mathrm{C}$ IV absorption profile around $z \approx 2.2$ galaxies using stacked background galaxy spectra and found that the mean C IV absorption strength falls off rapidly at projected distances of 50-100 kpc. Prochaska et al. (2013a) studied the CGM around $\mathrm{z} \approx 2$ bright quasars using closely projected foreground-background quasar pairs. They found that the CGM of quasar hosts exhibit significantly stronger C IV absorption as compared to $z \approx 2$ LBGs, especially at impact parameters greater than $100 \mathrm{kpc}$.

In this study, akin to the COS-Halos survey, we adopt a "galaxy selected" approach (Bordoloi et al. 2011; Tumlinson et al.2013). We first identify the projected galaxyquasar pairs, selected based on the galaxy properties, quasar brightness and the projected separation and then look for absorption in the rest frame of the galaxies. In the "blind" method, it is expensive to build up a statistically large sample of quasar-galaxy pairings with an impact parameter of $<100$ kpc. The galaxy selected approach allows us to probe the inner part of the CGM by design, enabling us to have better statistics at close impact parameters. Some of the difficulties that are encountered in correctly associating foreground galaxies with individual absorption systems (e.g. owing to limitations in the depth of the associated galaxy follow-up) are mitigated by designing a galaxy selected survey around a well defined set of foreground galaxies, rather than starting with a quasar spectrum and trying to identify nearby foreground galaxies responsible for the absorption.

The aim of this paper is to probe the CGM around $z<$ 0.1 low-mass galaxies, allowing us to systematically map the CGM traced by C IV gas out to their virial radii. Owing to their shallow potential wells, more intense radiation fields and lower metallicities, dwarf galaxies are believed to be inefficient at converting their gas content into stars (Schombert et al. 2001). Dwarf galaxies have higher gas fractions (ISM gas to stellar mass ratio) compared to high mass galaxies (Peeples et al. 2014) and because of their shallower potential wells (compared to the $L^{*}$ galaxies), this gas in principle can more easily be expelled into the CGM. Thus, such low-mass galaxies might possess a large reservoir of hidden mass in their CGM. In this work, we estimate the total CGM carbon mass, allowing us to extend the CGM metals census begun by COS-Halos (Peeples et al. 2014) to well below $L^{*}$.

This paper is organized as follows: Section 2 describes the design of the COS-Dwarfs survey. Section 3 describes the data collection and analysis methodology. Section 4.1 presents the 1-D and 2-D C IV radial absorption profiles. Section 4.2 presents the $C$ IV covering fraction estimates. Section 4.3 describes the dependence of C IV absorption strength on galaxy properties and Section 4.4 presents the C IV absorption kinematics. Section 5 presents the total carbon mass estimate in the CGM of these galaxies. Section 6 shows the expected incidence of C IV absorbers corresponding to sub- $\mathrm{L}^{*}$ galaxy halos. Section 7 compares several theoretical feedback prescriptions with observations. In section 8, we compare our results with the results from previous studies. Finally in Section 9 we summarize our conclusions.

Throughout our analysis we adopted a cosmology specified 


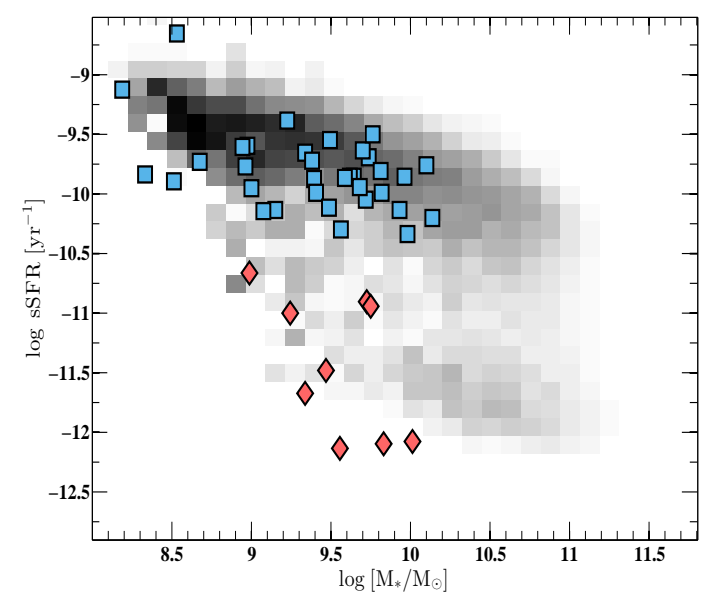

FIG. 1.- The galaxy color-magnitude diagram (sSFR vs $\mathrm{M}_{*}$ ) of all COSDwarfs galaxies. The blue squares and red diamonds represent star-forming and passive galaxies, respectively. The underlying distribution shows the sSFR vs $M_{*}$ for SDSS galaxies from (Schiminovich et al. 2007).

by $\Omega_{\mathrm{m}}=0.238, \Omega_{\Lambda}=0.762, \mathrm{H}_{0}=73.2 \mathrm{kms}^{-1} \mathrm{Mpc}^{-1}, \Omega_{\mathrm{b}}=$ 0.0416 . Distances and galaxy virial radii are given in proper coordinates.

\section{SURVEY MOTIVATIONS AND DESIGN}

Extending the COS-Halos survey to lower stellar mass can reveal how the CGM is configured and evolves as a function of stellar mass, but a new survey was required with design features that differ in many respects from COS-Halos. The requirement to select spectroscopically confirmed galaxies at $\log M_{*} / M_{\odot} \sim 8-9$ implied that the typical redshift needed to be lower than the median of $z=0.25$ in COS-Halos. Another consequence of the redshift range is that COS-Dwarfs galaxies could be selected on the basis of their SDSS spectroscopic redshifts (Abazajian et al. 2009), rather than photometric redshifts that were employed during the initial selection of the COS-Halos galaxies (Werk et al. 2012). Thus COS-Dwarfs was optimized to yield a sample of galaxies with $\log M_{*} / M_{\odot} \lesssim 10$ at $z \simeq 0.01-0.05$ using Ly $\alpha, \mathrm{C}$ IV and a range of other common low and intermediate ion diagnostics. The targeted sample consisted of 39 galaxies with QSO sightlines ranging out to $150 \mathrm{kpc}$ (physical), lying in front of UVbright QSOs at $z_{Q S O}=0.1-1$. In addition to these galaxies, we added four galaxies that were originally selected for COSHalos but were later omitted on the basis of low luminosity ${ }^{12}$. This yields a total of 43 galaxies that are used for this study. The sample comprises of the closest identified galaxy to each QSO sightline. No other galaxies with known redshifts from SDSS are closer than $300 \mathrm{kpc}$, though some of the sample galaxies have other galaxies at similar redshifts that are within $300-1000 \mathrm{kpc}$. We do not consider the sample biased with respect to galaxy neighbors, large-scale environment, or status as a satellite of a larger halo (in cases where neighbors are known). Throughout our analysis we assume that the closest galaxy - i.e. the one selected - is physically associated with the detected absorption.

The galaxy colors and stellar masses $M_{*}$ were derived from the ugriz SDSS photometry using the template-fitting approach implemented in the kcorrect code (Blanton \& Roweis

\footnotetext{
12 These galaxies were all selected for COS-Halos on the basis of photometric redshifts but were found by later spectroscopic measurements Werk et al. 2012) to have redshifts too low, and thus stellar masses too low, to be part of that sample. They are listed in Table 2 of Tumlinson et al. (2013).
}

2007) at the measured redshifts. Systematic errors from the mass-to-light ratio and IMF dominate the \pm 0.2 dex error in stellar mass (Werk et al. 2012). Star formation rates are estimated from the detected nebular emission lines or limited by their absence, with errors up to $\pm 50 \%$. Fiber losses are corrected using SDSS photometry similar to Werk et al. (2012). For passive galaxies the SFR is given as a $2 \sigma$ upper limit. Errors on combined quantities, such as the specific star formation rate $\left(\mathrm{sSFR}=\mathrm{SFR} / M_{*}\right)$, are obtained from quadrature sums of the basic error terms. The specific star formation rates (sSFR) and stellar masses $\left(\mathrm{M}_{*}\right)$ of the 43 galaxies used in this study are shown in Figure 1

The halo masses $M_{\text {halo }}$ are computed by interpolating along the abundance matching relation of Moster et al. (2010) at the stellar mass of the galaxy. We scale the projected distance from the sightline to the center of the host galaxy (impact parameter, $\mathrm{R}$ ) to the virial radius of the galaxy, approximated here as $R_{200}$, the radius at which the halo mass density is 200 times the critical matter density of the universe. This is given as

$$
\mathrm{R}_{200}^{3}=3 \mathrm{M}_{\text {halo }} / 4 \pi \Delta_{\text {vir }} \rho_{\text {matter }}
$$

where $\rho_{\text {matter }}$ is the critical density at the galaxy redshift times $\Omega_{\mathrm{m}}$, and $\Delta_{\mathrm{vir}}=200$. At the typical redshifts of the COSDwarfs galaxies $(z \sim 0.025), \mathrm{R}_{200}$ is slightly larger than the virial radius by a factor of $\sim 1.2$. Systematic errors in the stellar mass estimates and the scatter and uncertainty in the $\mathrm{M}_{\text {halo }}$ - $\mathrm{M}_{*}$ relation gives an uncertainty in $\mathrm{R}_{200}$ of approximately $50 \%$. Throughout this work we refer to this quantity $R_{200}$ as $\mathrm{R}_{\mathrm{vir}}$, as is commonly done. We refer the reader to Werk et al. (2012) for detailed methodology of measuring galaxy properties. All the relevant galaxy properties are tabulated in Table 1.

\section{DATA COLLECTION AND ANALYSIS}

The data reduction and analysis procedures for the present study follow the procedures described by Meiring et al. (2011) and Tumlinson et al. (2011a). In short, we obtain line measurements from 1D spectra that have been merged from individual exposures with different COS UV gratings and central wavelengths. The summation is done in counts per oversampled bin, once the different sub-exposures have been shifted into alignment based on common Milky Way interstellar absorption lines. The photocathode grid wires above the COS microchannel plates, casting shadows onto the detector are the main source of fixed-pattern noise in our data. Smaller fluctuations caused by the microchannel plate pores generally do not appear at the $\mathrm{S} / \mathrm{N}$ ratios of our data. There are other fixed-pattern noise features that must also be removed. We adopted flat-field reference files prepared and communicated to us by D. Massa at STScI and filtered for high-frequency noise by E. Jenkins. These $1 \mathrm{D}$ files allow us to correct the shadowed pixels by modifying the effective exposure time and count rate in each pixel prior to coadding it with the others. These flats do not correct for depressions in COS spectra caused by gain sag in some regions of the detector after prolonged exposure to bright geocoronal emission lines (Sahnow et al.2011). These gain-sag features can mimic real absorption lines, but the affected regions are flagged by the CALCOS pipeline (Holland et al. 2012). To avoid creating bogus absorption features, we reject the flagged gainsag regions in the coaddition process. The resulting $1 \mathrm{D}$, flatcorrected spectra are binned to Nyquist sampling with two bins per resolution element and a $\mathrm{S} / \mathrm{N}$ of $\sim 10-12$ per $\mathrm{COS}$ 
resolution element $\left(\mathrm{FWHM} \simeq 18 \mathrm{~km} \mathrm{~s}^{-1}\right)$. The fully reduced spectra are in units of counts per second with appropriate errors from counting statistics (Poisson) and errors propagated from the CALCOS (Holland et al. 2012) calibration steps.

With fully reduced spectra, we proceed with a semiautomated framework developed for COS-Halos to identify and measure the absorption lines associated with the foreground galaxies. We refer the reader to Tumlinson et al. (2013) for a detailed description of the method and only briefly summarize the steps here. We shift the reduced, coadded quasar COS spectrum to the rest-frame of the foreground galaxy, setting $v=0 \mathrm{~km} \mathrm{~s}^{-1}$ to the systemic redshift of the foreground galaxy. The systemic redshifts of the galaxies are measured to high precision $\left(\sigma_{\text {specz }} \sim 30 \mathrm{~km} \mathrm{~s}^{-1}\right.$ in the rest-frame) and we can focus on common lines at predictable places in observed wavelength. A detected absorption line is considered to be associated with a galaxy if it is within \pm $600 \mathrm{~km} \mathrm{~s}^{-1}$ of the systemic redshift of the galaxy. Short slices of data within $\pm 600 \mathrm{~km} \mathrm{~s}^{-1}$ of the systemic redshift of the galaxy are extracted around each line of interest, including the C IV doublet and Ly $\alpha$, CII 1334, SiIII 1206, SiIV 1393, SiIV 1402. Each slice is independently continuum-normalized using a fifth-order Legendre polynomial, and the equivalent width and column density of the absorption is measured (as a detection or an upper limit for non-detections) using the apparent optical depth (AOD) method of Savage \& Sembach (1991). For the key ion C IV, we visually inspect each doublet to confirm their presence and search for contamination, and to set the velocity range for AOD integration. To minimize contamination from other intervening absorption line systems or from the foreground ISM of the Milky Way, we attempt to identify every detection feature within $\mathrm{a} \pm 600 \mathrm{~km} \mathrm{~s}^{-1}$ range at the position of the C IV absorption doublet. Most such features are not associated with the target galaxy and are positively identified HI or metal lines associated with other absorbers at different redshifts along the line of sight. We confirm the presence of the C IV absorption doublet by excluding any alternate identification for the doublet and by looking at their apparent optical depth profiles. We accept apparent optical depth ratios ranging from 2:1 to $1: 1$ and require that the C IV absorption is aligned within $\pm 200 \mathrm{~km} \mathrm{~s}^{-1}$ of Ly $\alpha$ and other detected metal lines in that absorption system.

In addition to the AOD-derived column densities, we fit Voigt profiles to both absorption lines of the C IV doublet, to assess kinematic component structure and to estimate column densities for severely blended lines. The profile fitting improves on direct line integration of column densities using information about line shape and placement to constrain the fit. It also helps assess line saturation by taking into account the true line-spread function (LSF) of the instrument. We refer the reader to Tumlinson et al. (2013) for a detailed description of the Voigt profile fitting method. To summarize, we use an IDL iterative fitting program using the MPFIT software to optimize the fit and to estimate the errors on the fits. We fit column density $N$, Doppler $b$ parameter, and velocity offset $v$ for each component simultaneously for each C IV doublet. The initial parameters, including the number of components to be fitted, are set after visual examination of the data. The best fit parameters are obtained by performing $\chi^{2}$ minimization around $\pm 600 \mathrm{~km} \mathrm{~s}^{-1}$ of the foreground galaxy systemic redshift using the Levenberg-Marquardt algorithm. The detected $\mathrm{C}$ IV absorption lines and the corresponding Voigt profile fits are shown in the Appendix (Figure 11). C IV absorption at these redshifts are detected on the G160M grating of the COS spectrograph. For each individual quasar spectrum, we compute the $\mathrm{S} / \mathrm{N}$ and $3 \sigma$ detection threshold at the $\mathrm{C}$ IV rest frame. The quoted $3 \sigma$ detection thresholds are the mean uncertainty in measuring the $\mathrm{C}$ IV rest frame equivalent width with a 100 $\mathrm{km} \mathrm{s}^{-1}$ velocity window, within $\pm 600 \mathrm{~km} \mathrm{~s}^{-1}$ of the C IV line. All the measurements and galaxy properties are summarized in Table 1

\section{RESULTS}

In the following sections we discuss the variation of C IV absorption with different properties of the foreground galaxies, examining the radial profile, the dependence on galaxy sSFR, and absorber kinematics.

\subsection{The Spatial Distribution of C IV Absorption Around Galaxies}

To estimate the spatial extent of C IV absorption around these galaxies, we examine how the $\mathrm{C}$ IV rest-frame equivalent width $\left(W_{r}\right)$ and column density $\left(\mathrm{N}_{\mathrm{CIV}}\right)$ depend on the impact parameters $(\mathrm{R})$ and the virial radii $\mathrm{R}_{\mathrm{vir}}$ of the host galaxies. Figure 2 shows the 1-D C IV absorption profile around 43 galaxies as a function of $\mathrm{R}$ (left panels) and $\mathrm{R}_{\mathrm{vir}}$ (right panels). C IV absorption is seen around 17 galaxies (filled symbols), and no absorption is detected around 26 galaxies (open symbols). A galaxy is defined to be star-forming (blue squares) if its specific star formation rate (sSFR) is greater than $\log$ sSFR $\geq-10.6$.

Figure 2 reveals that the strongest C IV absorption is detected at smaller impact parameters. The CGM around these galaxies shows a drop in C IV absorption beyond a radius of $\sim$ $110 \mathrm{kpc}\left(\gtrsim 0.5 \mathrm{R}_{\mathrm{vir}}\right.$ ), (see also Liang \& Chen 2014 for a similar finding). All $\log N_{C I V} \geq 14$ absorption lines are detected within $\approx 90 \mathrm{kpc}$ of the galaxies. We parametrize the radial fall off in C IV absorption by characterizing the $1 \mathrm{D}$ radial profile in Figure 2 in terms of a power law with an exponential fall off. This characterization is given as

$$
\left\langle\mathrm{W}_{\mathrm{r}}(\mathrm{R})\right\rangle=\mathrm{W}_{0} \times\left(\frac{\mathrm{R}}{\mathrm{R}_{\mathrm{x}}}\right)^{-1} \times \exp \left(\frac{-\mathrm{R}_{\mathrm{vir}}}{\mathrm{R}_{\mathrm{x}}}\right)
$$

Here the characteristic radius $\mathrm{R}_{\mathrm{x}}$ sets the power of the exponential fall off for a galaxy with a given $\mathrm{R}_{\mathrm{vir}}$. We perform a maximum likelihood fit to the data to obtain the best fit parameters as $R_{x}=105 \pm 27 \mathrm{kpc}$ and, $\mathrm{W}_{0}=0.6 \pm 0.3 \AA$. The fitted mean absorption profile with $\left\langle\mathrm{R}_{\mathrm{vir}}\right\rangle=203 \mathrm{kpc}$ is the dashed red line in the top left panel of Figure 2

Figure 2 further reveals that the C IV absorption is patchy even at low impact parameters. At $\mathrm{R} \leq 60 \mathrm{kpc}, 53 \%$ (9 out of 17) of galaxies are associated with C IV absorption while $40 \%$ of the entire sample (17 out of 43) is associated with C IV absorption $\left(W_{r} \geq 90 \mathrm{~m} \AA\right)$. Even after excluding three nondetections with > $90 \mathrm{~m} \AA 3 \sigma$ detection thresholds (Table 1), we find that $43 \%$ of the entire sample (17 out of 40 ) is associated with C IV absorption. The patchiness is also evident in the scatter of C IV absorption strength for the detected absorbers ( standard deviation $=208 \mathrm{m \AA}$ ).

For star-forming galaxies, the COS-Dwarfs data clearly shows a trend between virial radius $\left(\mathrm{R}_{\mathrm{vir}}\right)$ and $\mathrm{C}$ IV absorption strength, that is quite similar to that observed for $\mathrm{Mg}$ II absorption (e.g. Chen et al. 2010, Bordoloi et al. 2011, Werk et al. 2013). As we probe the innermost CGM at small virial radii, increasingly stronger C IV absorbers are detected. This is different than the radial profile observed for 

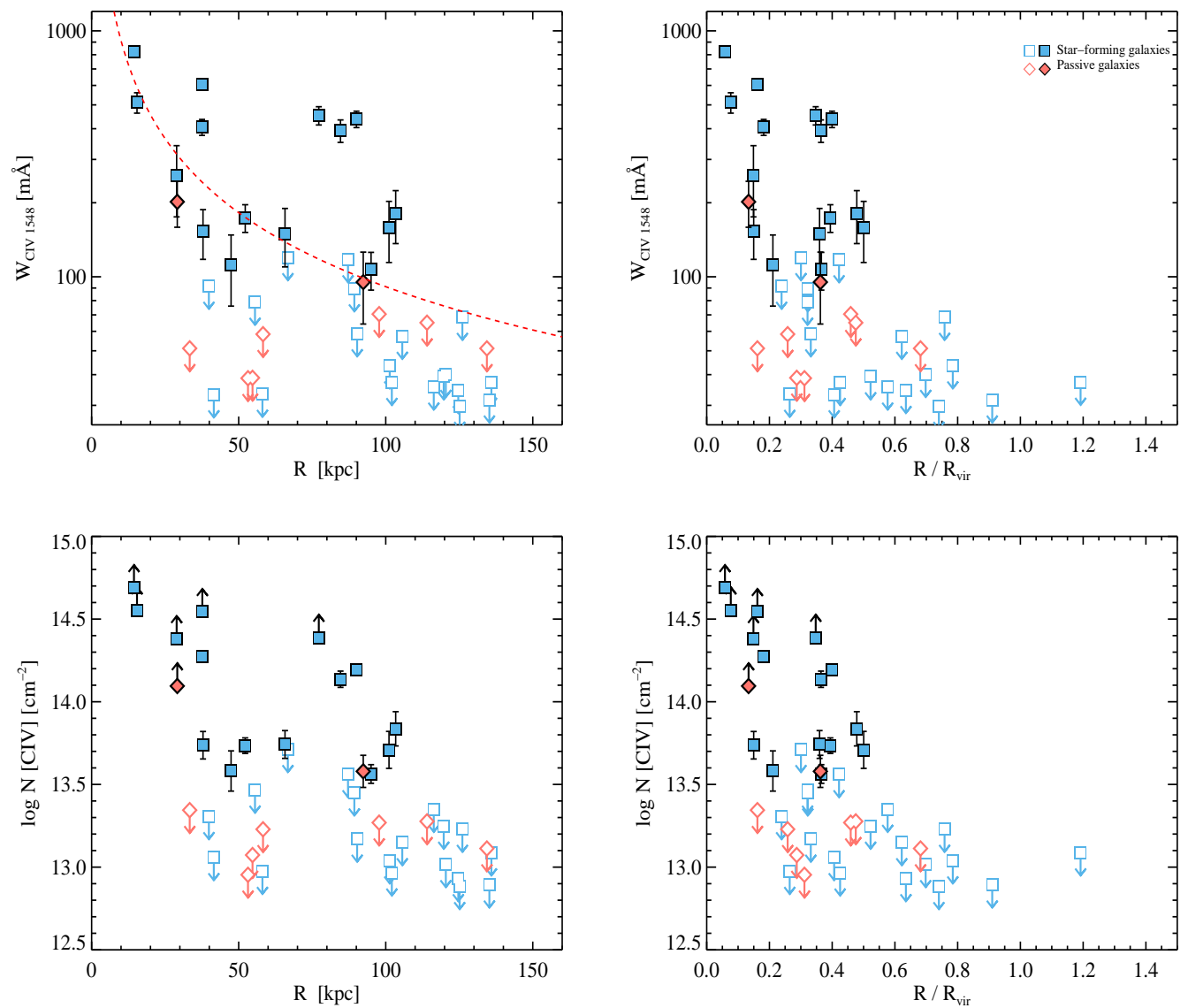

FIG. 2.- The 1-D C IV absorption profile around the COS-Dwarfs galaxies, in terms of R (left panels) and $\mathrm{R}_{\mathrm{vir}}$ (right panels). The blue squares and red diamonds represent star-forming and passive galaxies respectively. The filled symbols indicate detections while the open points with arrows indicate $2 \sigma$ upper limits of non-detections. Absorption strength declines with increasing projected galactocentric radius and no C IV absorption is detected beyond R $\gtrsim 110 \mathrm{kpc}$. The dashed red line represents the best fit radial profile to the data given by equation 2 Bottom Panels show the C IV absorption radial profile in terms of AOD column densities. The filled symbols with upward arrows are lower limits on C IV column densities.
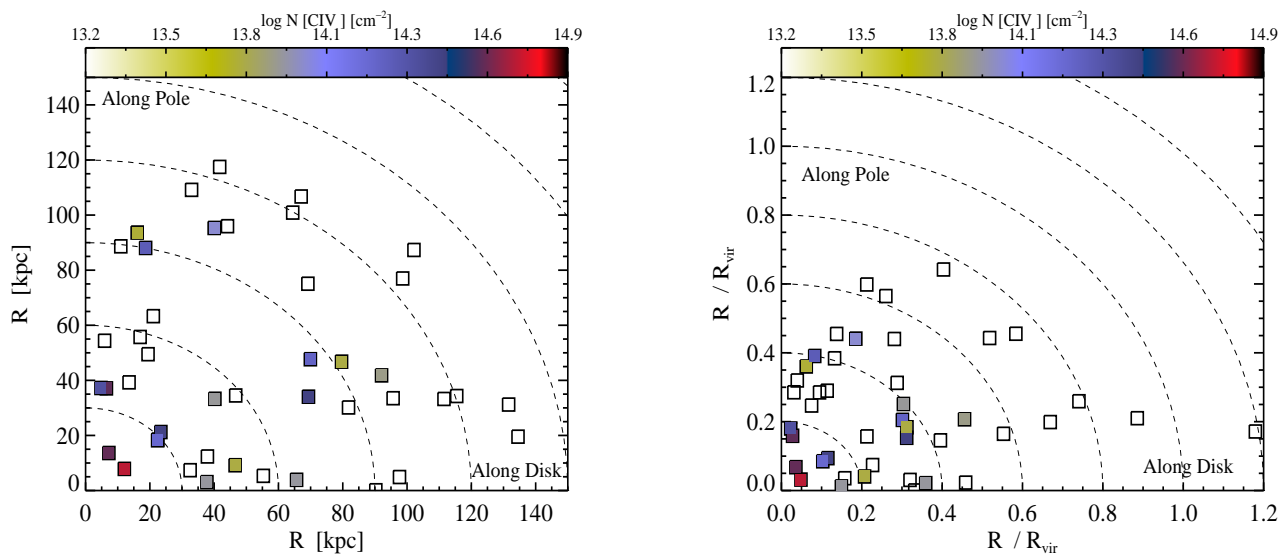

FIG. 3.- The projected 2-D C IV absorption radial profile around the COS-Dwarfs galaxies, in terms of R (left panel) and $\mathrm{R}_{\mathrm{vir}}$ (right panel). The open squares indicate non-detections and the filled squares are detections. The detections are color coded to reflect their AOD column densities. The points lying along the $\mathrm{y}$-axis represent lines of sight passing along the projected minor axis of the galaxy and the points lying along the $\mathrm{x}$-axis represent lines of sight passing along the projected major axis of the galaxy.

O VI (Tumlinson et al. 2011b), where the O VI radial profile for star-forming galaxies is flat out to $150 \mathrm{kpc}$ and there are very few non-detections. The observed C IV absorption profile is also not consistent with the O VI absorption profile observed around $>0.1 L^{*}$ galaxies at $z<0.2$ (Prochaska et al. 2011b). They detected extended ( $\approx 200 \mathrm{kpc}$ ) O VI absorption around $>0.1 L^{*}$ galaxies with high covering fraction $(\approx 80 \%)$. However, Prochaska et al. (2011b) found that, their small sample of dwarf galaxies $\left(<0.1 L^{*}\right)$, exhibit much lower $\mathrm{O}$ VI covering fraction for $\mathrm{R}>50 \mathrm{kpc}$ at $\langle z\rangle \approx 0.04$. It should 

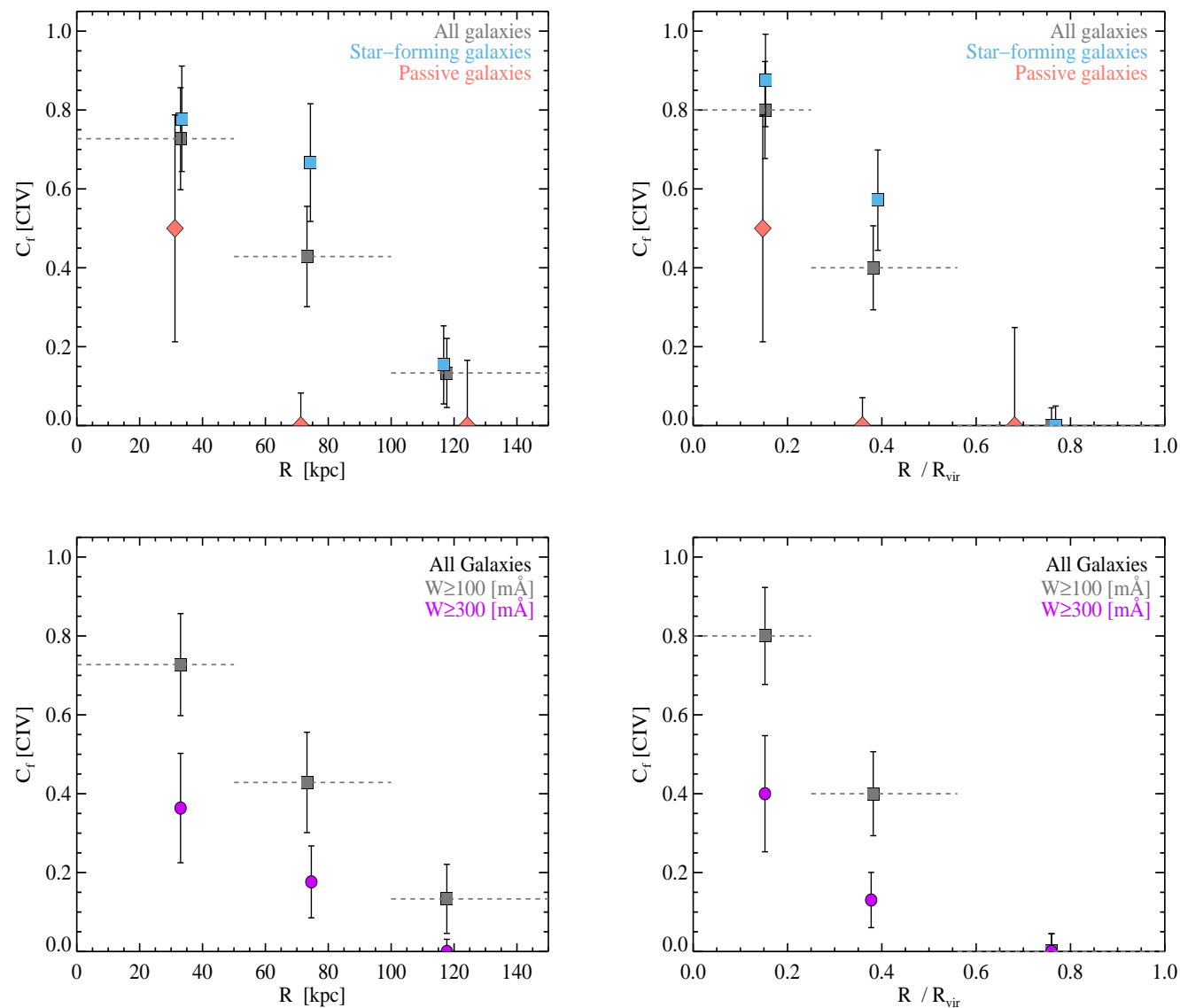

FIG. 4.- The C IV absorption covering fraction around galaxies, in terms of $\mathrm{R}$ (left panels) and $\mathrm{R}_{\mathrm{vir}}$ (right panels). The error bars on covering fraction represent the $68 \%$ confidence intervals. The horizontal dashed lines are range bars indicating the width of the radial bins. Top panels: The blue squares and red diamonds are covering fraction estimates for star-forming and passive galaxies, respectively, while the gray squares are covering fractions for all galaxies with $W \geq 100$ $\mathrm{m} \AA$. The covering fraction drops sharply beyond $0.2 R_{\text {vir }}$ and is zero at $R_{\text {vir }} \gtrsim 0.5$. Even very close to the galaxies, the C IV covering fraction is not unity. Bottom Panels: Covering fraction for C IV absorption around galaxies for $W \geq 100 \mathrm{~m} \AA$ (gray squares) and $W \geq 300 \mathrm{~m} \AA$ (blue circles), respectively.

be noted that $\mathrm{O}$ VI and $\mathrm{C}$ IV have different ionization potentials, and hence they trace CGM gas with different physical conditions. In particular, $\mathrm{O}$ VI is believed to trace more diffuse gas in the CGM compared to C IV. Part of the discrepancy between the $\mathrm{C}$ IV and O VI radial profiles could owe to different species tracing gases with different physical conditions. However, some absorbers show a remarkable kinematical correlation of the velocity centroids and line widths of species ranging from $\mathrm{H} \mathrm{I}, \mathrm{Mg}$ II, and C II up to O VI and NeVIII (Tripp et al. 2008, 2011; Meiring et al.2013; Savage et al. 2014). In these absorbers, models indicate that the low and high-ionization species must arise in different phases, but somehow the different phases are forced to move together and likely originate in the same general spatial region. Unfortunately, most current low-z absorber samples do not provide simultaneous coverage of $\mathrm{C}$ IV and O VI, but in a small number of cases where C IV and O VI are both observed, they are often found to be well-aligned and have essentially identical kinematics (Tripp et al. 2006; Savage et al. 2014). Thus, the different radial profiles from COS-Dwarfs and COS-Halos could alternatively be an intrinsic difference between sub- $\mathrm{L}^{*}$ and $L^{*}$ galaxies.

\subsubsection{Two-dimensional C IV absorption profile}

Since these low redshift galaxies have well resolved light profiles from ground based imaging, we use SDSS $r$ band pho- tometric measurements for each foreground galaxy to study the azimuthal dependence of C IV absorption. We compute the azimuthal angle $(\phi)$, the projected angle that the quasar line of sight makes with the projected major axis of the galaxy, by using SExtractor (Bertin \& Arnouts 1996) to estimate the position angle and the axis ratio. The azimuthal angles are presented in Table 1. The sense of the azimuthal angle is such that any sightline passing along the projected major axis of the galaxy is assigned $\phi=90^{\circ}$ and any sight line passing along the projected minor axis of the galaxy is assigned $\phi=0^{\circ}$.

Figure 3 shows the projected 2-D C IV absorption profile around the COS-Dwarfs galaxies in terms of $\mathrm{R}$ and $\mathrm{R}_{\mathrm{vir}}$ respectively. The open squares are the non-detections and the filled squares are color coded to reflect their absorption strengths. Visual inspection of Figure 3 shows that stronger absorbers have a marginal preference to be aligned towards the minor axis of the galaxies, however this trend is not statistically significant. A two sample KS test does not rule out the null hypothesis that the C IV absorbers are spherically distributed around the galaxies at $10 \%$ significance. Comparing the azimuthal asymmetry of C IV and Mg II absorption line systems (Bordoloi et al. 2011; Bordoloi et al. 2014) will allow us to constrain the geometry of the multi phase CGM in a unique way. The strong Mg II absorbers are preferentially seen along the projected minor axis of disk galaxies. However, if the observed C IV detections represent any azimuthal 
asymmetry, we would need $\approx 110$ absorber-galaxy pairs to rule out the null hypothesis that $\mathrm{C}$ IV absorption is uniformly distributed around the galaxies at $1 \%$ significance.

\subsection{IV Covering Fraction Estimates}

In the previous subsection, we have shown how the C IV absorption strength changes as we probe the CGM away from the galaxy. We have also shown that the number of nondetections increases as we probe higher projected distances. Here we quantify the observed incidence of $\mathrm{C} \mathrm{IV}$ absorption around galaxies in terms of covering fraction $\left(C_{f}\right)$. The covering fraction is defined as

$$
C_{f}=\frac{N_{W \geq W_{c u t}}(R)}{N_{t o t}(R)},
$$

where, $N_{W \geq W_{\text {cut }}}(R)$ is the number of lines of sight within a given radial bin from the galaxies, having associated absorbers of strength $W$ greater than some cutoff equivalent width $\left(W_{\text {cut }}\right)$. $N_{\text {tot }}(R)$ is the total number of lines of sight in that same radial bin.

The typical detection threshold for the COS-Dwarfs survey is $\approx 50-100 \mathrm{~m} \AA$. Throughout the paper, we will compute covering fraction only for absorption with $W_{\text {cut }} \geq 100 \mathrm{~m} \AA$. If we exclude the three non-detection with $3 \sigma$ detection threshold $>100 \mathrm{~m} \AA$ from the sample (Table 1 ), $C_{f}$ for the complete sample is $40 \%$ (16 detections out of 40 ).

We first evaluate $C_{f}$ for all star-forming and passive galaxies including all absorbers with $W_{\text {cut }} \geq 100 \mathrm{~m} \AA$. In Figure 4 we show the $C_{f}$ estimates as a function of $\mathrm{R}$ (top left panel) and $\mathrm{R}_{\mathrm{vir}}$ (top right panel). Star-forming galaxies (blue squares) exhibit a statistically higher covering fraction than passive galaxies (red diamonds). The gray squares represent the $C_{f}$ estimates for all galaxies. The error bars are $68 \%$ confidence intervals on the $C_{f}$ estimates. At close projected galactocentric distances the star-forming galaxies have very high covering fractions $(\approx 80 \%)$ that decrease to $\sim 65 \%$ at $\mathrm{R} \sim$ $80 \mathrm{kpc}$ or $\mathrm{R}_{\mathrm{vir}} \sim 0.4$. For $\mathrm{R}>100 \mathrm{kpc}$ the covering fractions are very low (15\%) and, in terms of $\mathrm{R}_{\mathrm{vir}}, C_{f}$ goes to zero as we probe beyond $\mathrm{R}_{\mathrm{vir}} \gtrsim 0.5$. The horizontal dashed lines are range bars indicating the span of the radial bins used to compute the covering fractions.

We further explore how $C_{f}$ varies for a different values of $W_{c u t}$ in the bottom panels of Figure 4. The $C_{f}$ estimates as a function of $\mathrm{R}$ (bottom left panel) and $\mathrm{R}_{\mathrm{vir}}$ (bottom right panel) are shown for $W_{\text {cut }} \geq 300 \mathrm{~m} \AA$ (purple circles) and $W_{\text {cut }} \geq 100$ $\mathrm{m} \AA$ (gray diamonds), respectively. At close galactocentric radius, the $C_{f}$ for "strong" C IV absorption $\left(W_{\text {cut }} \geq 300 \mathrm{~m} \AA\right)$ is about half ( $\sim 40 \%$ ) of the $C_{f}$ for absorbers with $W_{\text {cut }} \geq 100$ $\mathrm{m} \AA$. In both cases, the radial fall off in $C_{f}$ with distance exhibits a similar trend.

The C IV absorption is seen out to $\sim 110 \mathrm{kpc}$ in these galaxies $\left(\approx 0.5 \mathrm{R}_{\mathrm{vir}}\right.$ in terms of virial radius), beyond which $\mathrm{C}$ IV absorption is not detected in any of the sightlines ( 0 out of 11). This suggests that the C IV covering fraction falls off sharply at $0.5 \mathrm{R}_{\mathrm{vir}}$, beyond which there is little strong C IV absorption (see also Liang \& Chen 2014). This sharp cutoff is quite similar to that seen in MgII absorption line systems (Chen et al. 2010; Bordoloi et al. 2011; Nielsen et al. 2013).

\subsection{Dependence of C IV absorption with galaxy sSFR}

To characterize how C IV absorption depends on host galaxy properties, we study the variation of C IV absorption strength with specific star formation rate (sSFR). In Figure 5. the left panels show the variation of $\mathrm{C}$ IV equivalent width (top left) and AOD C IV column density (bottom left) with the sSFR of the COS-Dwarfs galaxies. We restrict this plot to include lines of sight passing within $0.5 \mathrm{R}_{\mathrm{vir}}$ because we only detect $\mathrm{C}$ IV absorption in these sight lines (with a typical detection threshold of $\approx 50-100 \mathrm{m \AA}$ ). We find that for the 24 galaxies that are identified as star-forming, there are 15 detections of C IV absorption (detection probability $\mathrm{P}=0.63 \pm 0.096$ at $68 \%$ confidence), while for the 8 passive galaxies there are two C IV detections (detection probability $\mathrm{P}=0.25 \pm 0.14$ at $68 \%$ confidence). If we exclude the three non-detections with a $3 \sigma$ detection threshold $>100 \mathrm{~m} \AA$ (all star-forming galaxies), the C IV detection probability for star-forming galaxies increases to $\mathrm{P}=0.71 \pm 0.1$ at $68 \%$ confidence interval. This suggests that the probability of detecting C IV absorption around a star-forming galaxy is generally higher than around a passive galaxy.

We test the statistical significance of the correlation of C IV absorption strength with host galaxy sSFR, by performing a generalized Kendall's tau test. We only consider absorber-galaxy pairs within $\mathrm{R}_{\mathrm{vir}} \leq 0.5$. We obtain $\tau=0.35$ (significance $\mathrm{P}=0.048$ ) and hence can reject the null hypothesis that there is no correlation between $\mathrm{C}$ IV absorption equivalent width and host galaxy sSFR at the $95 \%$ confidence level. We select all the galaxies within $\mathrm{R}_{\mathrm{vir}} \leq 0.5$ and perform a two-sample KS test that rules out the null hypothesis that the star-forming and passive galaxies are drawn from the same parent distribution of equivalent widths at $98 \%$ confidence level $(2.3 \sigma)$. We interpret these results as tentative, but not conclusive evidence that there is a direct relationship between C IV absorption strength and sSFR.

The correlation between galaxy star formation with stellar mass could potentially influence this apparent correlation found above. This can hinder our ability to conclusively identify star formation as the key influence on the presence of C IV . To assess whether star formation and not simply stellar mass has a direct relationship with the observed C IV, we select galaxies with $9 \leq \log \mathrm{M}_{*} / \mathrm{M}_{\odot} \leq 10$. In this range, there are 22 star-forming and 7 passive galaxies. We perform a two-sample KS test that rejects the null hypothesis that the star-forming and passive galaxies draw from the same parent distribution of equivalent widths at $>99.5 \%$ confidence level $(2.8 \sigma)$. This result supports our interpretation that there is tentative, but not conclusive evidence for a direct relationship between C IV absorption strength and sSFR.

The variation of C IV absorption strength with sSFR is further shown by adding the sample of Borthakur et al. (2013) (green squares), and the three COS-Halos galaxies for which we have C IV coverage (Figure 5 right panels). The COSHalos galaxies are marked with open circles for identification. These are primarily $L^{*}$ galaxies and four out of the five Borthakur et al. (2013) galaxies with detected C IV absorption are star-burst galaxies at high impact parameters. Combining the two samples, we find that out of the 33 galaxies that are identified as star-forming, there are 21 detections of C IV absorption (detection probability $\mathrm{P}=0.64 \pm 0.08$ at $68 \%$ confidence) and for the 18 passive galaxies there are three $\mathrm{C}$ IV detections (detection probability $\mathrm{P}=0.17 \pm 0.09$ at $68 \%$ confidence).

This suggests that at these equivalent width/column den- 

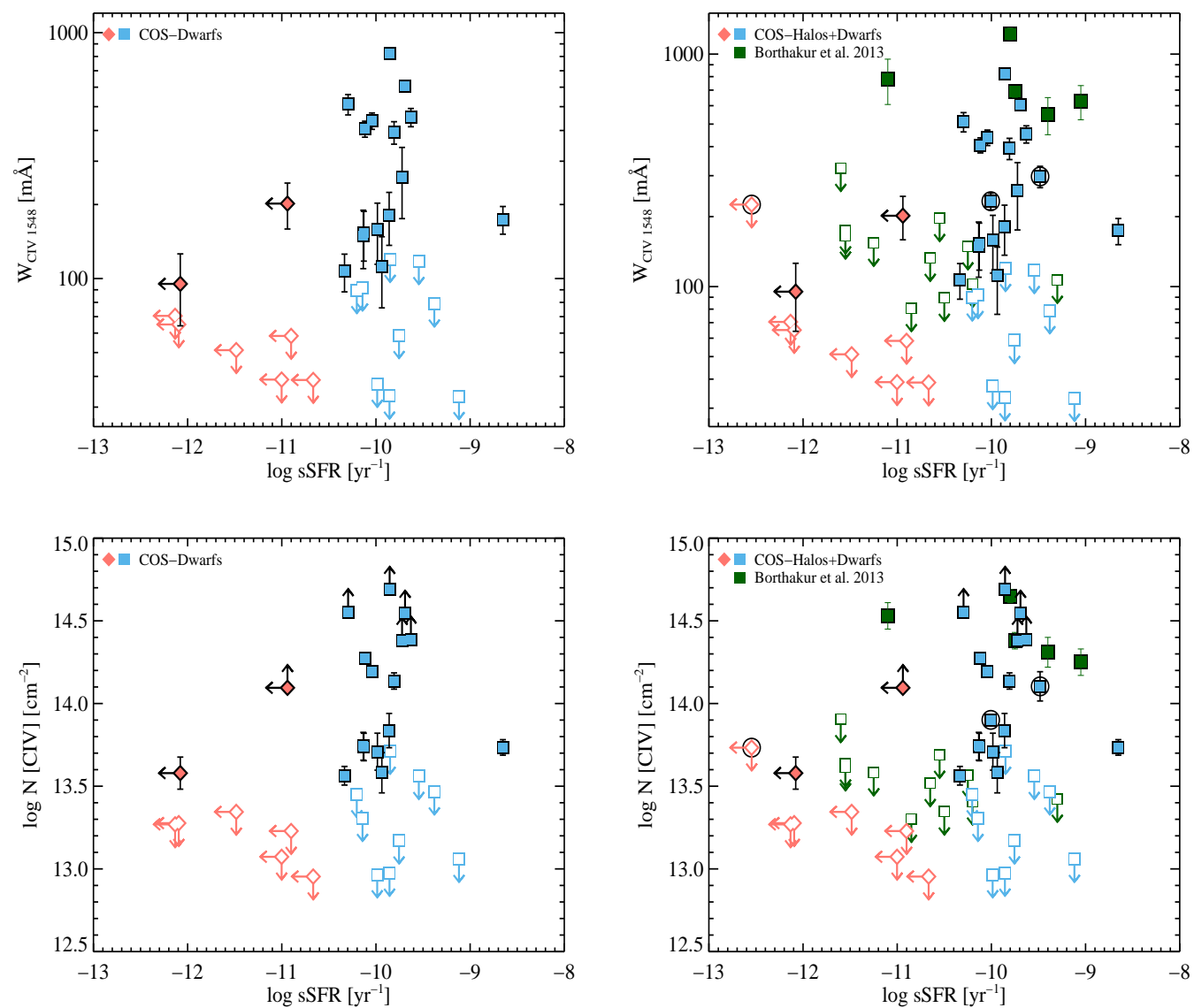

FIG. 5.- Top Panels: Dependence of C IV rest frame equivalent width on the sSFR of the host galaxies within $\mathrm{R}_{\mathrm{vir}} \leq 0.5$ from the COS-Dwarfs survey (left panel). The right panel shows the same after including the data from COS-Halos (tagged with open circles) and, Borthakur et al. (2013) (green points). The starforming (blue squares) and passive (red diamonds) galaxies show distinctly different C IV detection rates. The filled symbols represent detected C IV absorption and the open symbols with arrows are $2 \sigma$ upper limits on non-detections. Bottom Panels: Same as the top panels, now in terms of AOD column densities. The open symbols with downward arrows are $2 \sigma$ upper limits on non-detections and the filled symbols with upward arrows are lower limits on column densities.

sity limits, if a strong $\left(W_{r} \gtrsim 100 \mathrm{~m} \AA\right) \mathrm{C}$ IV absorption system is observed, it is more likely to be associated with a star-forming galaxy. One possible explanation for this high $\mathrm{C}$ IV detection rate amongst star-forming galaxies, along with the low covering fraction of C IV absorption beyond $0.5 \mathrm{R}_{\mathrm{vir}}$, could be that the C IV absorption is driven into the CGM by star-formation driven winds (Weiner et al. 2009; Rubin et al. 2012; Bordoloi et al. 2013) and they are falling back into the galaxy as a galactic fountain with a turn around radius of $\approx$ $0.5 \mathrm{R}_{\mathrm{vir}}$.

\subsection{IV Kinematics}

In this section we present the kinematics of the observed C IV absorption profiles and discuss whether the observed absorption is consistent with being bound to the dark matter halo of the host galaxy. We obtain the C IV column densities and kinematics by Voigt profile fitting as described in Section 3. Figure 6 shows the velocity centroids for each Voigt profile fitted component as a function of the stellar mass and the inferred dark matter halo mass for star-forming (blue squares) and passive (red diamonds) galaxies. The range bars indicate the velocity spread of each C IV absorption line system that were used to compute the equivalent widths of these systems. These are equivalent to the full width at zero optical depth. The full velocity width includes contributions from both thermal broadening and bulk flow and this is a proxy

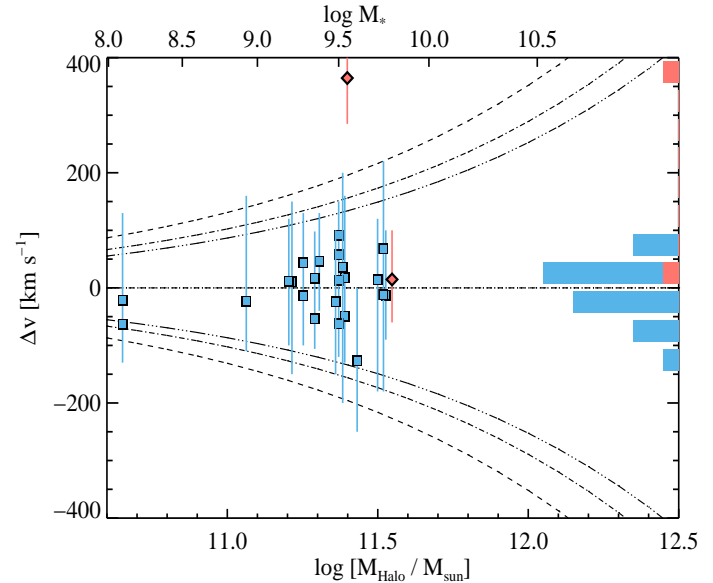

FIG. 6.- The C IV component velocity centroids with respect to the galaxies' systemic redshifts as a function of the inferred dark matter halo mass for star-forming (blue squares) and passive (red diamond) galaxies, respectively. The range bars indicate the maximum projected kinematic extent of C IV absorption for each system. The histogram represents the distribution of individual component velocities. The dashed lines show the mass-dependent escape velocities at $\mathrm{R}=50,100$ and $150 \mathrm{kpc}$, respectively.

for the maximum possible (projected) kinematic extent of the absorption. The distribution of the component velocities are clustered around the systemic velocity of the host galaxy, with a median velocity of $13 \mathrm{~km} \mathrm{~s}^{-1}$ and a standard deviation of 50 
$\mathrm{kms}^{-1}$. There is one exception, with one system being at $\approx$ $350 \mathrm{~km} \mathrm{~s}^{-1}$ from its host galaxy. However, statistically most of the detected C IV absorption is closely associated with the galaxies in velocity space. This is perhaps not surprising, since we are selecting lines of sight very close to the foreground galaxies. But there is no observational reason or systematic error or selection effect that prevents us from more commonly detecting strong C IV absorption at high velocities relative to the systemic redshift of the associated galaxies.

Figure 6] also compares these velocities with the escape velocities of the halos in which they reside. We convert the stellar masses of the galaxies to the total dark matter halo mass by using the method described in Moster et al. (2010). We assume a spherically symmetric NFW profile (with concentration parameter, $\mathrm{c}=15$ ) and calculate the escape velocity as a function of halo mass at three different radii $(\mathrm{R}=50,100,150$ kpc respectively). In Figure 6 these mass-dependent escape velocities are shown as dashed lines. We see that little of the fitted absorption velocity centroids exceed the estimated escape velocities of these galaxies. Some of the velocity ranges are comparable to the escape velocities (range bars) but that can be attributed to the line wings. We conclude that most of the detected C IV absorption is consistent with being bound to the dark matter halos of their host galaxies.

There is always the chance that these galaxies have associated C IV absorption at higher relative velocities that remains unseen because it is below our detection limits. We can roughly estimate that any such high velocity absorption components should have a factor of $\approx 5$-10 lower column densities than the typical detected absorption (Figure 2 bottom panels), since the detections typically have column densities that are 5-10 times above the detection limits.

\section{MINIMUM MASS OF CGM CARBON}

One of the striking findings of the COS-Halos survey was that around $L^{*}$ galaxies, there is about as much oxygen (proxied by O VI) as found in the ISM of those galaxies (Tumlinson et al. 2011b). Analysis of the lower ionization transitions further showed the presence of a large reservoir of metals and gas in the cool CGM (conservatively, $\mathrm{M}_{\mathrm{CGM}}^{\mathrm{cool}}>10^{9} \mathrm{M}_{\odot} ;$ Werk et al. 2014). This substantial metal budget helps in bridging the missing metals budget around $L^{*}$ galaxies (Peeples et al. 2014). The COS-Dwarfs survey is well suited to estimate the carbon mass in the CGM around sub-L* galaxies. The range of physical conditions in diffuse gas that can contain significant amounts of C IV is narrow enough that we can obtain robust lower limits on the total mass of carbon in the CGM of dwarf galaxies.

The total C IV mass encompassed within an impact parameter $(\mathrm{R})$ is given as,

$$
\mathrm{M}_{\mathrm{CIV}}=\pi \mathrm{R}^{2}\left\langle\mathrm{~N}_{\mathrm{CIV}}\right\rangle 12 \mathrm{~m}_{\mathrm{H}} \mathrm{C}_{\mathrm{f}},
$$

where $\mathrm{C}_{\mathrm{f}}$ is the mean covering fraction of $\mathrm{C}$ IV absorption around galaxies within radius $\mathrm{R}$ and $\left\langle\mathrm{N}_{\mathrm{CIV}}\right\rangle$ is the mean $\mathrm{C}$ IV column density within $\mathrm{R}$. We obtain a robust lower limit on carbon mass $\left(M_{\text {carbon }}\right)$ by applying a conservative ionization correction to $M_{C I V}$. To estimate the ionization correction, we have considered the ionization state of C IV over a wide range of temperatures using the CLOUDY photoionization code (Ferland et al. 1998), assuming ionization equilibrium and including both collisional ionization and photoionization similar to Tumlinson et al. (2011b). Under typical CGM physical conditions, the ionization timescales are of the order of $\sim 10^{7-8}$ years or less, which is much smaller than the halo dynamical timescales of $10^{9}$ years. Hence it is unlikely that a large fraction of the CGM gas would be far from ionization equilibrium. In Figure 7 (left panel), the curves trace the fraction of gas-phase carbon in the C IV ionization state ( $f_{\mathrm{CIV}}$ ) as a function of temperature for four overdensities relative to the cosmic mean density $(\rho / \bar{\rho})$. At the highest overdensities $(\rho / \bar{\rho} \geq 1000$, black curve), collisional ionization dominates and for all lower overdensities, photoionization by the extragalactic background would cause the increase of $f_{C I V}$ at low temperatures. The blue band shows the expected carbon mass of the galaxies' ISM if they lie on the standard relation between $M_{I S M}$ and $M_{*}$ and follow the mass-metallicity relation $(\mathrm{MZR})$ at $\log \mathrm{M}_{*}=9.5$. Regardless of whether photoionization or collisional ionization dominates, we assume the most conservative measure of the ionization correction to estimate the carbon mass as $f_{C I V}=0.3$.

Scaling for values typical to our sample and applying a conservative C IV correction, we find that the minimum carbon mass for all galaxies is

$\mathrm{M}_{\text {carbon }} \gtrsim 1.2 \times 10^{6} \mathrm{M}_{\odot}\left(\frac{\mathrm{N}_{\mathrm{CIV}}}{10^{14} \mathrm{~cm}^{-2}}\right) \times\left(\frac{\mathrm{R}}{110 \mathrm{kpc}}\right)^{2} \times\left(\frac{0.3}{\mathrm{f}_{\mathrm{CIV}}}\right)$,

where we find that within $110 \mathrm{kpc}, 17$ out of 32 galaxies show C IV absorption. The covering fraction and mean column densities are measured in two radial bins: 8 out of 11 detections within $R<50 \mathrm{kpc}$ and 9 out of 21 detections within $50 \leq R \leq 110 \mathrm{kpc}$. The column densities used in equation 5 are derived from Voigt profile fitting and represent the entire range in stellar mass. The mean column density within $R<50 \mathrm{kpc}$ is $4 \times 10^{14} \mathrm{~cm}^{-2}$ and within $50 \leq R \leq 110 \mathrm{kpc}$ is $8.2 \times 10^{13} \mathrm{~cm}^{-2}$. We estimate $M_{\text {carbon }}$ using both Voigt profile fitting and AOD column densities. Carbon masses derived using both methods are tabulated in Table 2. The column densities derived using the AOD method are lower limits for saturated lines; therefore the carbon masses are only conservative lower limits. The column densities derived from Voigt profile fitting are more reliable, however for severely saturated lines, they are also probably underestimating the column densities.

If these galaxies lie on the stellar metallicity relation (Woo et al. 2008), the mean trend of gas fractions, and the gas-phase mass-metallicity relation (Peeples et al. 2014) for low-z galaxies, then they have interstellar carbon masses of $M_{I S M}^{C}=1.6 \times 10^{5}$ to $5 \times 10^{6} \mathrm{M}_{\odot}$ and masses of carbon in stars $\left(M_{\text {star }}^{C}\right)=10^{4}$ to $1.5 \times 10^{6} \mathrm{M}_{\odot}$, where we have assumed solar abundance ratios of $\mathrm{C} / \mathrm{Z}=0.18$ by mass (i.e., $[12+\log (C / H)]_{\odot}=8.50$ and $Z_{\odot}=0.0153$; Caffau et al. 2011). Figure 7 (right panel), shows the minimum CGM carbon mass of star-forming galaxies (green points), for $f_{C I V}=0.3$, compared with the interstellar carbon mass (blue band) and stellar carbon mass (red band) as a function of mass (Peeples et al. 2014). The range in the blue band denotes the uncertainty in the ISM carbon mass owing primarily to uncertainties in the calibration of the gas-phase metallicity indicators and partially to the uncertainties in the gas fractions. The narrower red band denotes systematic uncertainties introduced to the mass of carbon in stars from uncertainties in the overall solar abundance scale but does not take into account possible nonsolar abundance ratios. Our key finding is that the minimum CGM carbon mass is $50 \%$ to $80 \%$ of the total ISM carbon mass and is always higher than the total mass of carbon in stars. 

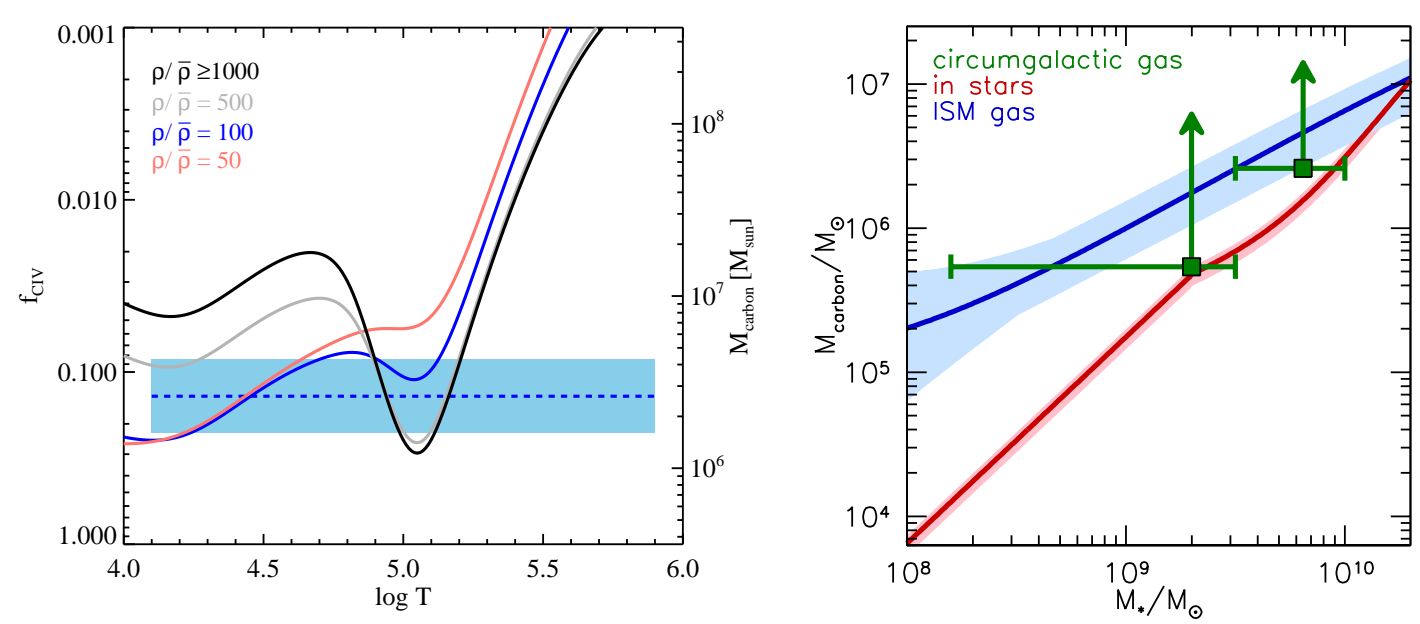

FIG. 7.- Carbon mass estimates in the CGM of sub-L* galaxies compared to their galactic reservoirs. Left Panel: The curves show the variation of the fraction of gas-phase carbon in the C IV ionization state $\left(f_{C I V}\right)$ with temperature for four overdensities relative to the cosmic mean $(\rho / \bar{\rho})$. For $\rho / \bar{\rho} \geq 1000$ (black curve), collisional ionization dominates. For the lower overdensities photoionization by the extragalactic background can increase $f_{C I V}$ at low temperatures. The blue band shows the expected carbon mass in the galaxies' ISM at $\log \mathrm{M}_{*}=9.5$, if the galaxies lie on the standard $M_{I S M}$ vs $M_{*}$ relation and follow the mass-metallicity relation (MZR). Right Panel: The CGM carbon mass (green points) compared to the interstellar carbon mass (blue band) and the carbon mass in stars (red band) as a function $M_{*}$. The green points show the conservative minimum carbon mass for blue galaxies in those mass ranges (Table 2].

The ratio of carbon mass in the ISM to the CGM remains more or less constant for the two different mass bins probed (assuming a constant ionization correction). It should be stressed that the CGM carbon masses quoted here are conservative lower limits, as we assume a limiting ionization correction of $f_{C I V}=0.3$ and the $\mathrm{C}$ IV column densities may be underestimated owing to saturation. For the typical densities expected at $\mathrm{R} \approx 100 \mathrm{kpc}, f_{C I V}$ exceeds 0.3 only at $\mathrm{T} \approx 10^{5.05} \mathrm{~K}$ and $f_{C I V}$ exceeds 0.05 at $\mathrm{T} \approx 10^{4.8}-10^{5.25} \mathrm{~K}$. Hence the total carbon mass can easily be even higher by a factor of 6 , higher than the ISM carbon mass in these galaxies. This result is analogous to the findings of COS-Halos oxygen metal budget (Tumlinson et al.2011b), where it was found that a significant mass of oxygen exists in the CGM of $L^{*}$ galaxies, and is comparable to the mass of oxygen in the ISM of those galaxies.

We estimate the carbon mass out to $110 \mathrm{kpc}$, beyond which no C IV absorption is detected. However, it is plausible that some diffuse C IV gas is present below the survey detection threshold, at larger radii. To ascertain how much carbon could be "hidden" beyond $110 \mathrm{kpc}$, we extend equation 5 out to $\approx$ $220 \mathrm{kpc}$. We assume a mean column density at $R>110 \mathrm{kpc}$ of $3 \times 10^{13} \mathrm{~cm}^{-2}$, which is comparable to our typical detection limits. We compute the total carbon within $220 \mathrm{kpc}$ and find that the total increase in carbon mass in going from $110 \mathrm{kpc}$ to $220 \mathrm{kpc}$ is $\approx 20 \%$. Hence the bulk of the carbon mass observed in the CGM should be observed within $110 \mathrm{kpc}$ (which corresponds to roughly $0.5 \mathrm{R}_{\mathrm{vir}}$ ) of the host galaxy.

\section{EXPECTED INCIDENCE OF C IV ABSORBERS}

In this section we estimate the expected redshift-path incidence of $\mathrm{C}$ IV absorbers exceeding a particular absorption threshold $W_{\text {cut }}$, around $\log M_{*} \leq 10^{10} \mathrm{M}_{\odot}$ galaxies at $z \approx 0$. This exercise is the opposite of one undertaken for blind QSO absorption line studies (e.g. Cooksey et al. 2010, Shull et al. 2014); where one takes the observed number of absorption systems per unit redshift and calculates the CGM cross section and number density required to account for the rate of incidence, assuming that the cross-section is all contributed by galaxies. Tumlinson \& Fang (2005) used the observed number density distributions of intergalactic $\mathrm{O}$ VI absorbers to constrain the metal distribution in the low- $z$ IGM, and

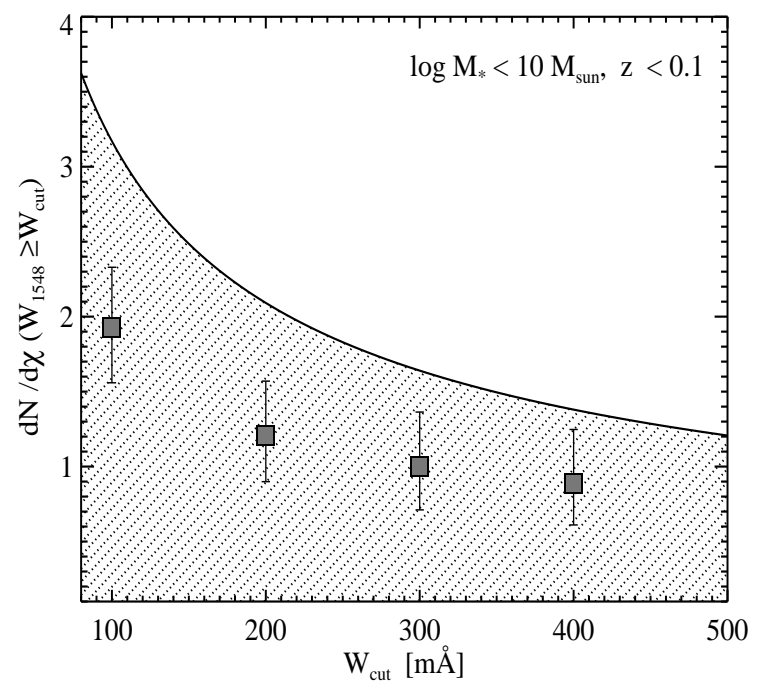

FIG. 8.- Cumulative absorber line density as a function of CIV 1548 equivalent width. The summed $d N / d \chi$ for COS-Dwarfs galaxies are shown as gray squares. The hashed region shows the cumulative $d N / d \chi$ at $\mathrm{z}<0.4$, adopted from Cooksey et al. (2010). COS-Dwarfs galaxies can account for $\sim 60 \%$ of all low-z C IV absorbers.

found that observed dN/dz of O VI absorbers can be explained by the extended CGM of sub-L*galaxies. Prochaska et al. (2011b) performed a similar calculation based on their analysis of $\mathrm{O}$ VI surrounding $\mathrm{z} \sim 0.1$ galaxies and concluded that the majority of O VI systems are associated with the extended CGM of sub-L*galaxies.

Here we infer the rate of incidence of C IV absorption around the COS-Dwarfs sample exceeding a particular absorption threshold $W_{\text {cut }}$ defined as,

$$
\frac{\mathrm{dN}}{\mathrm{d} \chi}\left(\mathrm{W}>\mathrm{W}_{\text {cut }}\right)=\frac{\mathrm{c}}{\mathrm{H}_{0}} \mathrm{C}_{\mathrm{f}} \mathrm{n}_{\text {gal }} \pi \mathrm{R}_{\max }^{2}
$$

where $\mathrm{C}_{\mathrm{f}}$ is the covering fraction of absorbers with (W $>\mathrm{W}_{\text {cut }}$ ) and $\mathrm{R}_{\max }$ is the maximum impact parameter at which an $\left(\mathrm{W}>\mathrm{W}_{\text {cut }}\right)$ absorber is observed. For COS-Dwarfs, we have a sample of galaxies at $z<0.1$ with a well defined 
stellar mass function (Moustakas et al. 2013). We integrate the double Schechter function fit to the $z<0.1$ mass distribution within $8 \leq \log M_{*} / M_{\odot} \leq 10$ and get a cumulative number density of COS-Dwarfs like galaxies $\mathrm{n}_{\text {gal }} \approx 10^{-1.39} \mathrm{Mpc}^{-3}$. These values translate to a rate of incidence at $W>100 \mathrm{~m} \AA$, $d N / d \chi \approx 1.93 \pm 0.4$, at $W>300 \mathrm{~m} \AA, d N / d \chi \approx 1.0 \pm 0.32$ and at $W>400 \mathrm{~m} \AA, d N / d \chi \approx 0.89 \pm 0.36$. The observed incidence of $\mathrm{C} I V$ systems at the same equivalent width threshold at $\mathrm{z}<0.4$ is reported to be for $(W>100 \mathrm{m \AA}) \approx 3.17$, for $(W>300 \mathrm{m \AA}) \approx 1.64$ and for $(W>400 \mathrm{~m} \AA) \approx 1.38$ (Cooksey et al. 2010). We present the cumulative absorber line density as a function of C IV equivalent width for COSDwarfs in Figure 8 . We adopt the C IV frequency distribution function at $z<0.4$, described in Cooksey et al. (2010), and it is integrated to infer the absorber line densities at different limiting equivalent widths. This is shown as the hashed region in Figure 8. It should be noted that all the line densities (and the corresponding error estimates) shown in Figure 8 are correlated. Comparing the line densities measured for COSDwarfs (gray squares) with the total cumulative absorber line densities (hashed regions) we argue that the low-mass galaxies having $8 \leq \log M_{*} / M_{\odot} \leq 10$ at $\mathrm{z}<0.1$, could easily account for almost $61 \%(\mathrm{~W}>100 \mathrm{m \AA}, \mathrm{W}>300 \mathrm{~m} \AA$ ), and $64 \%$ (W > $400 \mathrm{m \AA}$ ) of the strong C IV absorbers observed today. The remaining $\sim 30$ to $40 \%$ of the C IV absorbers can be accounted for by the $>L^{*}$ galaxies with a covering fraction of $\approx 40 \%$ with $R_{\max } \approx 200 \mathrm{kpc}$. It is plausible that $1 / 2-2 / 3$ of strong C IV in blind IGM samples arise in sub- $\mathrm{L}^{*}$ halos.

\section{COMPARISON WITH SIMULATIONS}

We use a set of cosmological GADGET-2 Nbody+smoothed particle hydrodynamical (SPH) simulations (Springel 2005) to compare with our observations of C IV covering fractions. The simulations have a periodic volume with a box length of $32 h^{-1} \mathrm{Mpc}$, contain $2 \times 512^{3}$ dark matter and gas particles, and use a $\Lambda$ CDM cosmology based on the WMAP 9-year results (Hinshaw et al. 2013). The gas particle mass is $4.5 \times 10^{6} \mathrm{M}_{\odot}$, the dark particle mass is $2.3 \times 10^{7} \mathrm{M}_{\odot}$, and the softening length is $\epsilon=1.25 h^{-1} \mathrm{kpc}$.

We run mock sight lines through three simulations exploring different prescriptions for galactic super-winds around galaxies chosen to match the stellar masses in passive and star-forming subsamples of COS-Dwarfs. These simulations were introduced by Davé et al. (2013), including the constant wind (cw), the energy-driven wind (ezw) and the no winds (NW) models. These wind models are defined by parameter choices of $\eta$, the mass loading factor of star formationdriven winds defined as $\eta \equiv \dot{M}_{\text {wind }} / \dot{M}_{\mathrm{SF}}$, and wind velocity, $v_{\text {wind }}$. The $\mathrm{cw}$ model uses a constant value of $\eta=2$ and $v_{\text {wind }}=680 \mathrm{~km} \mathrm{~s}^{-1}$ representing a $100 \%$ conversion efficiency of supernova energy from stars above $8 M_{\odot}$ to kinetic outflows. The ezw model uses energy-driven relations $\eta \sim \sigma^{-2}$ and $v_{\text {wind }} \sim \sigma$ below $\sigma=75 \mathrm{~km} \mathrm{~s}^{-1}$ and $\eta \sim \sigma^{-1}$ for higher $\sigma$. A quenching prescription described in Davé et al. (2013) is used in the ezw model, but does not affect the galaxy masses we explore for COS-Dwarfs. The no winds model has no outflows (i.e. $\eta=0$ ).

The spectral generator specexbin casts sight lines at impact parameters out to $150 \mathrm{kpc}$ around galaxies selected in each $z=0.025$ simulation output to match the COSDwarfs sample. The Haardt \& Madau (2001) ionization background is assumed but our results are indistinguishable if
Haardt \& Madau (2012) is used instead. We search around simulated galaxies matching the $M_{*}$ distribution of COSDwarfs galaxies divided into star-forming and passive samples based on the subdivision of $\mathrm{SSFR}=10^{-10.6} \mathrm{yr}^{-1}$. This sample results in different distributions of sSFR for each wind model compared to what is observed in COS-Dwarfs, with ezw providing the best match, because this wind model agrees best with the abundance matching (AM) constraints of Behroozi et al. (2013). In each case, we select isolated galaxies defined as not having another galaxy $\geq 50 \%$ as massive of the targeted galaxy's mass within $300 \mathrm{kpc}$.

In the $M_{*} / \mathrm{sSFR}$-selected sample, satellite galaxies fulfilling the isolation criteria are allowed in the sample, but make up only $4-9 \%$ of the simulated samples. Satellite galaxies are most often selected in the passive subsample around groups where $M_{\text {halo }}>10^{13} M_{\odot}$, because low-sSFR isolated galaxies are rare in the simulations. We cast 4 sight lines at 15 equallyspaced impact parameters ranging from 5 to $145 \mathrm{kpc}$ around three times as many galaxies as COS-Dwarfs, or 117 in total, for a total of 7020 mock sightlines per wind model. We then sum up the C IV $1548 \AA$ equivalent width within $\pm 600 \mathrm{~km} \mathrm{~s}^{-1}$ of the velocity of the galaxy.

Figure 9 shows the results of the COS-Dwarfs mock surveys compared to the actual observations. The left panels show the simulated galaxy parameters of $M_{*}$ and SSFR for each wind model: ezw (purple, top panels) and cw (green, bottom panels) with binned histograms, both compared to the COS-Dwarfs galaxies (gray histograms, red/blue squares). The simulated galaxies are required to have the COS-Dwarfs $M_{*}$ distribution within bins of 0.2 dex, but the simulated sSFR is constrained only to match the star-forming and passive samples using the division of $10^{-10.6} \mathrm{yr}^{-1}$. We note that ezw provides the best fit to the distributions of sSFR. The ezw feedback parameters were intentionally tuned to match the AM constraints of Behroozi et al. (2013) via the selection of the $\sigma=75 \mathrm{~km} \mathrm{~s}^{-1}$ threshold below which energy-driven scalings take effect for ezw. In contrast, cw winds are more efficient at suppressing star formation, and predict a comparatively constant $M_{*} / M_{\text {halo }}$ ratio with many more massive halos hosting centrals than any other wind model.

The middle panels show the simulated covering fractions of $100 \mathrm{~m} \AA$ and $300 \mathrm{~m} \AA \mathrm{C}$ IV as a function of impact parameter for each wind model, compared to the covering fractions from Section 4.2. Based on C IV covering fractions, ezw provides the best overall fits, although the ezw model over-estimates the extent of $100 \mathrm{~m} \AA \mathrm{C}$ IV detections (solid bands) at $>100$ kpc. The ezw model better reproduces the $C$ IV covering fraction for $300 \mathrm{~m} \AA \mathrm{C}$ IV detections (hashed band). The right panels show the C IV column density radial profiles of the starforming galaxies as compared to the simulations. The gray hashed regions represent the $1 \sigma$ spread of column density in the model sightlines.

The cw model performs poorly in reproducing both CGM and galaxy properties. At close impact parameters, the cw model systematically under predicts the C IV covering fractions and at high impact parameters, it over-predicts the extent of $100 \mathrm{~m} \AA \mathrm{C}$ IV covering fraction (solid band). Previous work has shown that this model predicts a stellar mass function that is too steep (Oppenheimer et al. 2010) and a presentday sSFR distribution that is too low for low-mass galaxies (Davé et al. 2011). A faster $v_{\text {wind }}$ arising from low-mass galaxies in $\mathrm{cw}$ relative to ezw results in a flatter dependence 

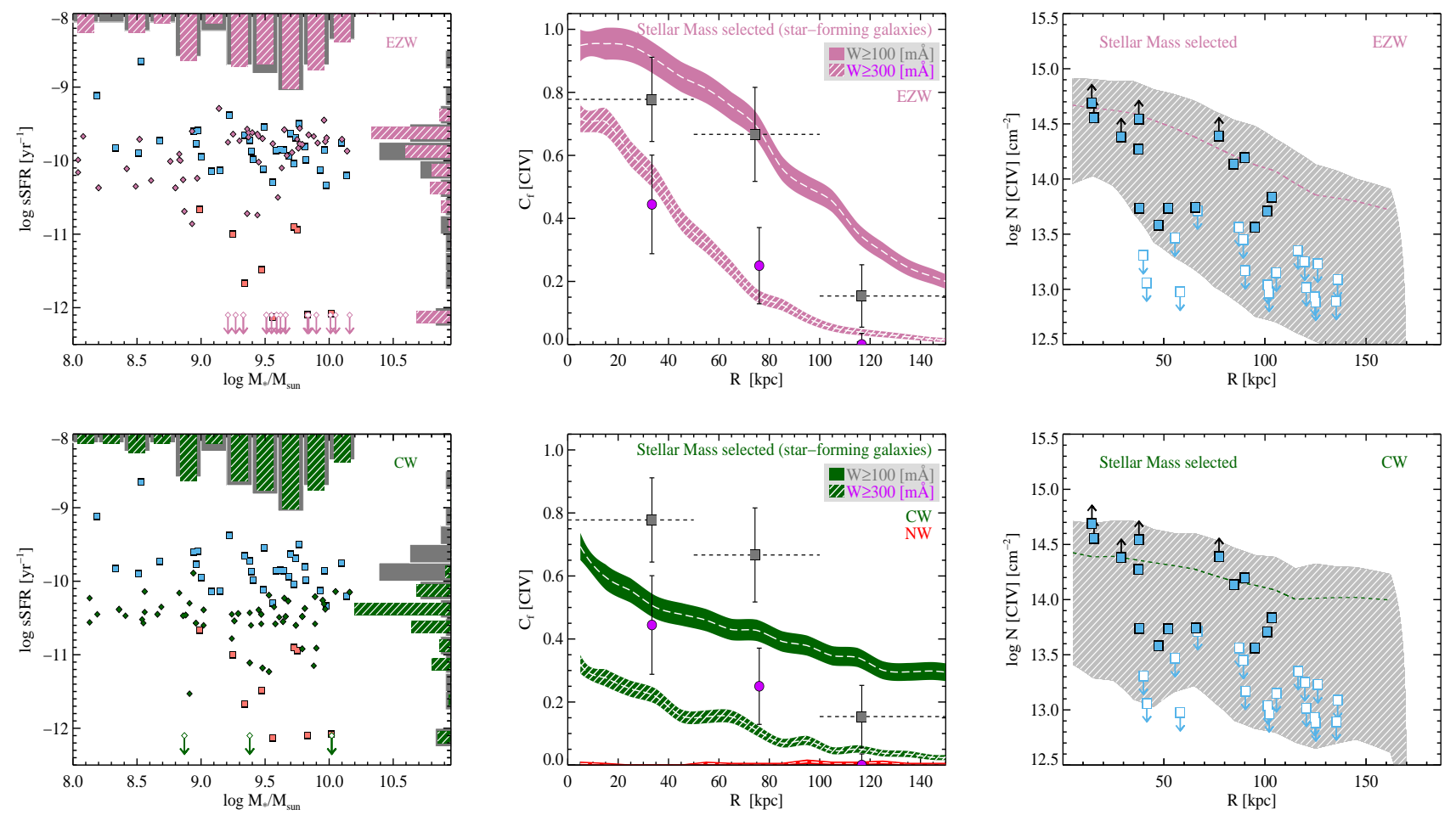

FIG. 9.- Comparison of different feedback prescriptions in hydro simulations with observations. The distribution of sSFR and stellar mass (left panels) for 117 galaxies with different feedback prescriptions (diamonds) and 43 COS-Dwarfs galaxies (blue and red squares) are shown respectively. The C IV covering fraction estimates for star-forming galaxies are shown in the middle panels. The purple and green bands represent covering fraction estimates for the two wind models and the red band represents same for the no wind model. The error bars represent the 68\% confidence intervals. Right Panels: The C IV column density radial profiles of the star-forming galaxies is compared to the simulations. The hashed regions represent the $1 \sigma$ spread of column density in the model sight lines. The dashed green and purple lines indicate the mean column density radial profiles in the simulations.

of C IV covering fractions as metals are more likely to be pushed to larger distances.

We compare the sSFR distributions of the ezw and the cw models with that of the COS-Dwarfs galaxies and find that for the ezw model, a two sample KS test cannot rule out the null hypothesis that the two sSFR distributions were drawn from the same parent sample at $>10 \%$ significance. For the cw model a two sample KS test rules out the null hypothesis that the two sSFR distributions are drawn from the same parent sample at $0.001 \%$ significance level. Further, we perform a likelihood ratio test to compare which model (ezw or cw) best represents the observed C IV covering fractions. We obtain a $\mathrm{P}$ value of $\approx 0.01$, which indicates that there is strong evidence that the ezw model represents the data better than the $\mathrm{cw}$ model. Hence, constraints from both the sSFR distribution and the $C$ IV covering fraction suggest that the ezw model better represents the observations as compared to the cw model.

We further compare the observed C IV covering fraction with that measured in simulations with no winds (NW) (Figure 9. red band middle panel). The NW model predicts C IV covering fractions of $\sim 1 \%$ to $2 \%$ at all impact parameters. Hence the metallic content of the CGM around sub-L*galaxies cannot be explained by tidal debris or rampressure stripping alone, as these are the only processes for distributing metals into the CGM in our NW simulations. In a recent study, Liang \& Chen (2014) also found that metals around low-mass galaxies are primarily concentrated within the inner virial radii of the galaxies. While they find similar observational trends within their sample, they conclude that winds are inefficient at these masses, but our quantitative comparison to hydrodynamic simulations with and without winds strongly indicates that strong outflows are necessary explain the observed C IV in the CGM.

In summary, our exploration of simulations yields the best fits for an ezw model that ejects cool gas $\left(T \sim 10^{4} \mathrm{~K}\right)$ at moderate velocities $\left(v_{\text {wind }}=150-300 \mathrm{~km} \mathrm{~s}^{-1}\right)$ and high massloading factors $(\eta=5-15)$. These winds enrich the local CGM where this metal-enriched gas can re-accrete back onto the galaxy and sustain the observed $z \sim 0$ sSFR distribution of low-mass galaxies.

Finally, if we select simulated central galaxies based on halo masses derived from the abundance matching applied to COS-Dwarfs, we would find more distinguishing power using C IV covering fractions between the various wind models, but at the cost of selecting distributions of $M_{*}$ and sSFR that do not match COS-Dwarfs. The ezw model does not change much because these galaxies agree well with abundance matching constraints, while $\mathrm{cw}$ would have far lower covering fractions and no detections of $300 \mathrm{~m} \AA \mathrm{CIV}$ absorbers, because the galaxies form less stars and the $v_{\text {wind }}=$ $680 \mathrm{~km} \mathrm{~s}^{-1}$ heat the CGM, suppressing star formation. These trends show that in the low-mass galaxy regime explored by COS-Dwarfs, our simulations find that covering fractions scale with galaxy $M_{*}$.

\section{COMPARISON WITH PREVIOUS STUDIES}

In this section, we present the combined measurements of previous studies from the literature, which characterized the C IV absorption profile around galaxies. We stress that this comparison involves galaxies with heterogeneous mass, SFR, and selection, so this is not a statistically rigorous comparison. 
In the top panels of Figure 10, we present the C IV absorption observed in the COS-Halos survey (two detections and one non-detection) and this work. The blue and red data points correspond to star-forming and passive galaxies, respectively. All the open symbols with arrows indicate $2 \sigma$ non-detections.

Using HST Key Project data, Chen et al. (2001) characterized the $\mathrm{C}$ IV absorption profile around $50 z \approx 0.4$ galaxies with background quasar lines of sight passing within 300 kpc of the host galaxies. In Figure 10, these measurements are represented as gray points in the top left and the middle left panels. They found C IV absorption out to $\mathrm{R} \sim 100$ kpc with abrupt boundaries between $\mathrm{C}$ IV absorbing and nonabsorbing regions. They also reported that the $\mathrm{C}$ IV absorption strength does not depend strongly on galaxy surface brightness, redshift, or morphological types. They found in their sample $28 \%$ (14 out of 50) of the galaxies are associated with C IV absorption.

In another study, Borthakur et al. (2013), targeted a sample of $20 z<0.2$ galaxies and constrained the C IV absorption in 17 of these galaxies within $\mathrm{R} \leq 200 \mathrm{kpc}$. They found that 4 out of 5 star-bursting galaxies exhibit strong C IV absorption out to $200 \mathrm{kpc}$. These are represented as green points in the top left and middle right panels of Figure 10.

At higher redshifts, Steidel et al. (2010) used stacked background galaxy spectra to characterize the C IV absorption around 512 foreground $\mathrm{z} \approx 2.2$ galaxies out to $125 \mathrm{kpc}$. They found that the C IV absorption strength falls off sharply between 50-100 kpc. In Figure 10, the purple data points in top left and middle right panels represent these measurements. In the Steidel et al. (2010) study, the spectral resolution was too low to resolve the C IV doublet. Hence the quoted C IV absorption strength was summed over both lines of the doublet. Here we use half of the quoted value in Steidel et al. (2010) to compare with the CIV 1548 absorption strength. Prochaska et al. (2013b) use a subset of 428 quasars that show Ly $\alpha$ absorption to probe the CGM around $\mathrm{z} \approx 2$ quasar host galaxies. They find that $\mathrm{C}$ IV absorption around quasar hosts extends farther than that observed around LBGs at the same redshifts and the covering fraction of strong $\mathrm{C}$ IV absorption extends beyond $200 \mathrm{kpc}$. We select all the C IV absorbers within $800 \mathrm{~km} \mathrm{~s}^{-1}$ of the systemic redshift of the quasar host galaxy and plot them in Figure 10 (orange points).

The top left panel of Figure 10 combines all of the above together. It should be noted that these galaxies represent a very heterogeneous sample of galaxies, spanning many decades in mass, varying star formation rates, and redshifts. Excluding the star-bursting systems of Borthakur et al. (2013) and the Prochaska et al. (2013b) study, the average C IV absorption profile remains quite unchanged from $z \approx 2.2$ (Steidel et al. 2010), to $\mathrm{z} \approx 0.4$ (Chen et al. 2001), all the way to $\mathrm{z} \sim 0$ (COS-Dwarfs). We can detect C IV absorption out to $100 \mathrm{kpc}$ for all these galaxies.

In the bottom panel of Figure 10, we present the C IV absorption covering fraction for the $z<0.5$ studies of COS-Halos, COS-Dwarfs, Borthakur et al. (2013) and Chen et al. (2001), as a function of impact parameter. The horizontal dashed lines are range bars indicating the width of the bins. We find that the $\mathrm{C}$ IV absorption covering fraction drops from $70 \%(\mathrm{~W} \geq 100 \mathrm{m \AA}$ ) at $\mathrm{R} \leq 50 \mathrm{kpc}$ to $30 \%$ at $\mathrm{R} \approx 100 \mathrm{kpc}$. The $1 \overline{5} \%$ covering fraction seen at extended radius of $\mathrm{R}>150 \mathrm{kpc}$ is primarily contributed by the starbursting systems of Borthakur et al. (2013). However, there are observations of individual C IV absorption line systems, where the host galaxy is observed to be at high impact parameters (Tripp et al. 2006; Burchett et al. 2013) and may indicate diverse origins of $\mathrm{C}$ IV absorbers from that described here. This combined dataset indeed suggests that for regular main-sequence non-starburst galaxies, C IV absorption can been detected out to $\approx 100 \mathrm{kpc}$ from $\mathrm{z} \sim 2$ to $\mathrm{z} \sim 0$ at these detection limits.

\section{CONCLUSIONS}

In this work, we have mapped the spatial distribution of C IV gas around a set of 43 galaxies having mass $M_{*} \leq 10^{10}$ $\mathrm{M}_{\odot}$ at $z<0.1$. The main results of this study are as follows.

- C IV absorption is detected out to $\approx 100 \mathrm{kpc}$ from the galaxies. C IV absorption strength drops off with projected galactocentric radius from the associated galaxy; beyond $0.5 \mathrm{R}_{\mathrm{vir}}$ no $\mathrm{C}$ IV absorption is detected at our sensitivity limits $(\sim 50-100 \mathrm{~m} \AA)$.

- The C IV absorption is patchy even at small impact parameters. At $\mathrm{R} \leq 60 \mathrm{kpc}, 9$ out of $17(60 \%)$ galaxies are associated with $\mathrm{C}$ IV absorption; at $\mathrm{R} \leq 150$ kpc 17 out of $40(43 \%)$ galaxies are associated with C IV absorption. The patchiness is also evident in the scatter of C IV absorption strength for the detected absorbers (standard deviation $=208 \mathrm{~m} \AA$ ).

- For star-forming galaxies, the covering fraction of $W \geq 100 \mathrm{~m} \AA$ CIV 1548 absorption is nearly unity at $\mathrm{R} / \mathrm{R}_{\mathrm{vir}} \leq 0.2$, falling off to $60 \%$ at $0.4 \mathrm{R} / \mathrm{R}_{\mathrm{vir}}$ and then to zero at $\mathrm{R} / \mathrm{R}_{\mathrm{vir}} \gtrsim 0.5$.

- We find that strong C IV absorbers are primarily detected around star-forming galaxies and report a correlation between the detected C IV absorption strength and sSFR of the host galaxies within $0.5 R_{\text {vir }}$. We find that within $0.5 \mathrm{R}_{\mathrm{vir}}$, the C IV detection probability around star-forming galaxies is $\mathrm{P}=0.63 \pm 0.096$ and around passive galaxies is $\mathrm{P}=0.25 \pm 0.14$. We reject the null hypothesis that there is no correlation between C IV absorption strength and host galaxy sSFR at the 95\% confidence level.

- The detected C IV absorbers are kinematically consistent with being bound to the dark matter halos of their host galaxies. The absorption centroids cluster around the systemic zero velocity of their host galaxies with a median velocity of $13 \mathrm{~km} \mathrm{~s}^{-1}$ and a standard deviation of $50 \mathrm{~km} \mathrm{~s}^{-1}$.

- We estimate the minimum carbon mass in the CGM of these galaxies, and find that there is at least $\gtrsim$ $1.2 \times 10^{6} \mathrm{M}_{\odot}$ of carbon within $110 \mathrm{kpc}$ of these galaxies. This is comparable to the total carbon mass in the ISM of these galaxies, and more than the total carbon mass contained in the stars of these galaxies. Amongst star-forming galaxies, the CGM around low mass galaxies $\left(8 \leq \log \mathrm{M}_{*} / \mathrm{M}_{\odot} \leq 9.5\right)$ can have a carbon mass of at least $\gtrsim 0.5 \times 10^{6} \mathrm{M}_{\odot}$, and around $9.5 \leq \log \mathrm{M}_{*} / \mathrm{M}_{\odot} \leq 10$ galaxies the carbon mass is at least $\gtrsim 2.6 \times 10^{6} \mathrm{M}_{\odot}$. This is a conservative lower limit, and the CGM carbon mass could easily be factor of six higher. 

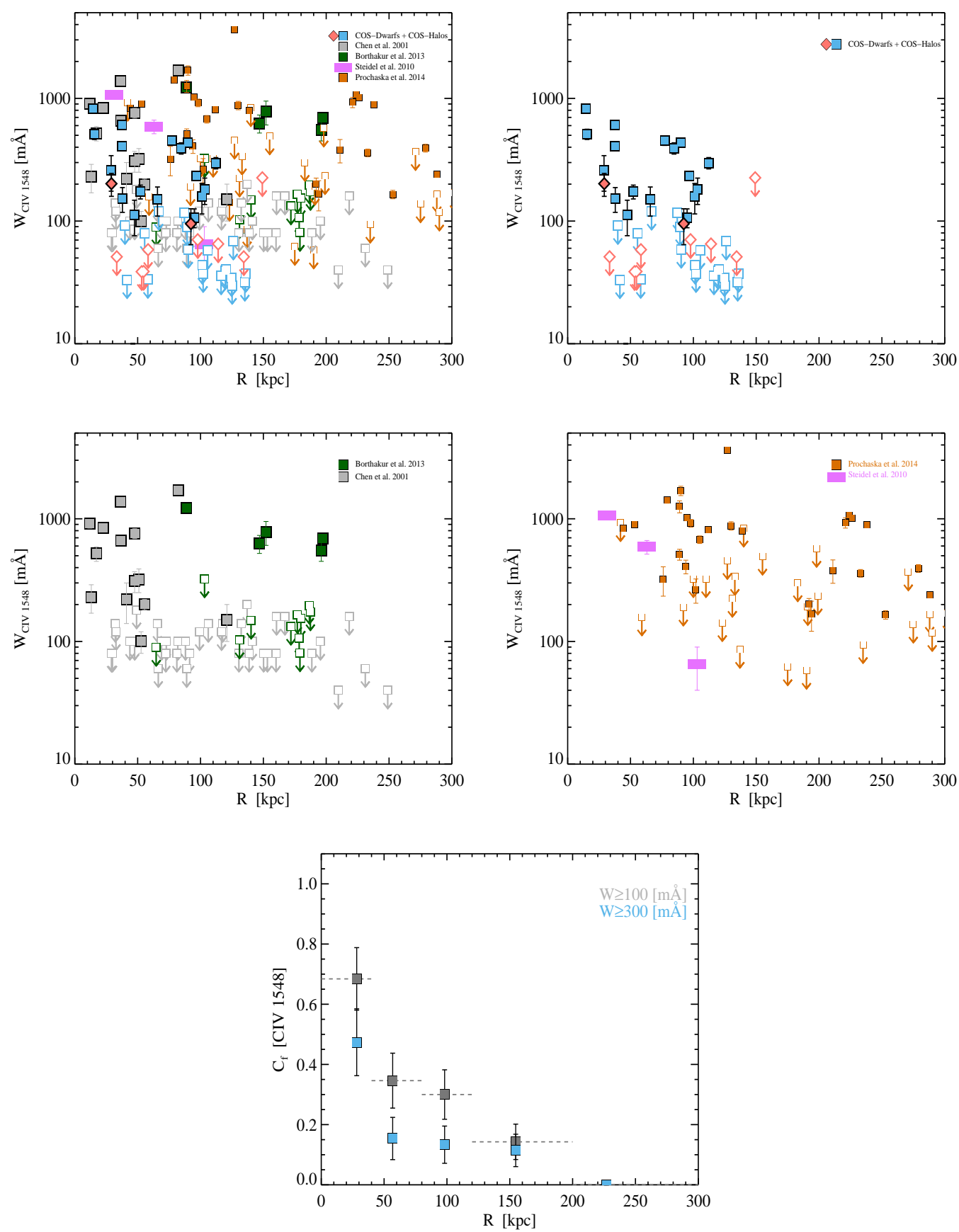

FIG. 10.- C IV absorption profile compiled from available literature data These galaxies are not constrained in terms of their masses, star-formation rates, and redshifts. Top Four Panels:- 1-D C IV absorption profile as a function of $\mathrm{R}$ from the literature. The blue and red points are star-forming and passive galaxies from the COS-Dwarfs (this work) and COS-Halos (Tumlinson et al. 2013) survey. The gray points are from Chen et al. (2001) at $\mathrm{z} \sim 0.4$ and green points are from Borthakur et al. (2013) at $\mathrm{z}<0.2$. The purple points are data from stacked spectra analysis of (Steidel et al. 2010) at $\mathrm{z} \approx 2.2$ and orange points are data from (Prochaska et al. 2013b) mapping the CGM around quasar host galaxies at $\mathrm{z} \approx 2$. All the open arrow symbols are $2 \sigma$ non detections. Bottom Panel:- The 1-D covering fraction profile as a function of $\mathrm{R}$ of all the $z<0.5$, literature data with $\mathrm{W} \geq 100 \mathrm{~m} \AA$ (gray squares) and with $\mathrm{W} \geq 300 \mathrm{~m} \AA$ (blue squares). The error bars represent the $68 \%$ confidence intervals. Horizontal dashed lines are range bars indicating the width of the radial bins.

- Combining the cosmic number density of the galaxies in our sample from the galaxy stellar mass function, with the observed C IV absorption cross-section could account for $\approx 60 \%$ of all intervening $\mathrm{C}$ IV absorption with (W > $100 \mathrm{~m} \AA$ and $\mathrm{W}>300 \mathrm{~m} \AA$ ) and $\approx 64 \%$ (W > $400 \mathrm{~mA}$ ) $\mathrm{C}$ IV absorbers in the low- $z$ Universe, observed along QSO lines of sight.
- We compare the observed and simulated galaxies at the same stellar mass in three flavors of feedback models in hydrodynamical simulations. Comparing the hydrodynamical simulations with and without winds, we conclude that the observed metallic content of the CGM around sub-L* galaxies cannot be explained by tidal debris and ram pressure stripping alone, and strong outflows are required to explain the observations. We find 
that the energy-driven wind model (ezw) is a better overall fit to the present star formation rates and CGM C IV covering fraction measurements as compared to the constant velocity wind model $(\mathrm{cw})$.

In summary, we examined the CGM of sub-L*galaxies traced by C IV absorption. The detection of CGM carbon masses (traced by C IV) comparable to the ISM carbon mass of these low mass galaxies suggest that a substantial fraction of the baryon budget might be hidden in the CGM of these galaxies. In future work, we shall focus on the other ionization species to characterize the CGM of these sub- $\mathrm{L}^{*}$ galaxies and extend the baryon census began by the COS-Halos survey to these low masses.

\section{ACKNOWLEDGEMENT}

Support for program GO12248 was provided by NASA through a grant from the Space Telescope Science Institute, which is operated by the Association of Universities for Research in Astronomy, Inc., under NASA contract NAS 526555. BDO was supported by HST grant HST-AR-12841. Support for the simulations presented in this work was provided by the Ahmanson Foundation.

\section{REFERENCES}

Abazajian, K. N., et al. 2009, ApJS, 182, 543

Adelberger, K. L., Shapley, A. E., Steidel, C. C., Pettini, M., Erb, D. K., \& Reddy, N. A. 2005, ApJ, 629, 636

Adelberger, K. L., Steidel, C. C., Shapley, A. E., \& Pettini, M. 2003, ApJ, 584,45

Behroozi, P. S., Wechsler, R. H., \& Conroy, C. 2013, ApJ, 770, 57

Bertin, E. \& Arnouts, S. 1996, A\&AS, 117, 393

Blanton, M. R. \& Roweis, S. 2007, AJ, 133, 734

Blanton, M. R., et al. 2001, AJ, 121, 2358

Bordoloi, R., Lilly, S. J., Kacprzak, G. G., \& Churchill, C. W. 2014, ApJ, 784, 108

Bordoloi, R., et al. 2011, The Astrophysical Journal, 743, 10

Bordoloi, R., et al. 2013, ArXiv e-prints :1307.6553

Borthakur, S., Heckman, T., Strickland, D., Wild, V., \& Schiminovich, D. 2013, ApJ, 768, 18

Burchett, J. N., Tripp, T. M., Werk, J. K., Howk, J. C., Prochaska, J. X., Ford, A. B., \& Davé, R. 2013, ApJL, 779, L17

Caffau, E., Ludwig, H.-G., Steffen, M., Freytag, B., \& Bonifacio, P. 2011, Sol. Phys., 268, 255

Chen, H., Helsby, J. E., Gauthier, J., Shectman, S. A., Thompson, I. B., \& Tinker, J. L. 2010, ApJ, 714, 1521

Chen, H.-W., Lanzetta, K. M., \& Webb, J. K. 2001, ApJ, 556, 158

Cooksey, K. L., Kao, M. M., Simcoe, R. A., O’Meara, J. M., \& Prochaska, J. X. 2013, ApJ, 763, 37

Cooksey, K. L., Thom, C., Prochaska, J. X., \& Chen, H.-W. 2010, ApJ, 708, 868

Danforth, C. W. \& Shull, J. M. 2008, ApJ, 679, 194

Danforth, C. W., et al. 2014, ArXiv e-prints: 1402.2655

Davé, R., Finlator, K., \& Oppenheimer, B. D. 2011, MNRAS, 416, 1354

Davé, R., Katz, N., Oppenheimer, B. D., Kollmeier, J. A., \& Weinberg, D. H. 2013, MNRAS, 434, 2645

D’Odorico, V., Calura, F., Cristiani, S., \& Viel, M. 2010, MNRAS, 401, 2715

Ferland, G. J., Korista, K. T., Verner, D. A., Ferguson, J. W., Kingdon, J. B., \& Verner, E. M. 1998, PASP, 110, 761

Green, J. C., et al. 2012, ApJ, 744, 60

Haardt, F. \& Madau, P. 2001, in Clusters of Galaxies and the High Redshift Universe Observed in X-rays, ed. D. M. Neumann \& J. T. V. Tran

Haardt, F. \& Madau, P. 2012, ApJ, 746, 125

Hinshaw, G., et al. 2013, ApJS, 208, 19

Holland, S. T. et al. 2012, Cosmic Origins Spectrograph Instrument Handbook for Cycle 21 v. 5.0

Liang, C. J. \& Chen, H.-W. 2014, ArXiv e-prints:1402.3602

Lilly, S. J., et al. 2009, ApJS, 184, 218

Meiring, J. D., Tripp, T. M., Prochaska, J. X., Tumlinson, J., Werk, J., Jenkins, E. B., Thom, C., O’Meara, J. M., \& Sembach, K. R. 2011, ApJ, 732,35

Meiring, J. D., Tripp, T. M., Werk, J. K., Howk, J. C., Jenkins, E. B., Prochaska, J. X., Lehner, N., \& Sembach, K. R. 2013, ApJ, 767, 49

Moster, B. P., Somerville, R. S., Maulbetsch, C., van den Bosch, F. C., Macciò, A. V., Naab, T., \& Oser, L. 2010, ApJ, 710, 903

Moustakas, J., Coil, A. L., Aird, J., Blanton, M. R., Cool, R. J., Eisenstein, D. J., Mendez, A. J., Wong, K. C., Zhu, G., \& Arnouts, S. 2013, ApJ, 767, 50

Nielsen, N. M., Churchill, C. W., \& Kacprzak, G. G. 2013, ApJ, 776, 115
Oppenheimer, B. D., Davé, R., Kereš, D., Fardal, M., Katz, N., Kollmeier, J. A., \& Weinberg, D. H. 2010, MNRAS, 406, 2325

Peeples, M. S., Werk, J. K., Tumlinson, J., Oppenheimer, B. D., Prochaska, J. X., Katz, N., \& Weinberg, D. H. 2014, ApJ, 786, 54

Prochaska, J. X., Hennawi, J. F., \& Simcoe, R. A. 2013a, ApJL, 762, L19

Prochaska, J. X., Weiner, B., Chen, H.-W., Mulchaey, J., \& Cooksey, K. 2011a, ApJ, 740, 91

Prochaska, J. X., Weiner, B., Chen, H.-W., Mulchaey, J., \& Cooksey, K. 2011b, ApJ, 740, 91

Prochaska, J. X., et al. 2013b, ApJ, 776, 136

Rubin, K. H. R., Prochaska, J. X., Koo, D. C., \& Phillips, A. C. 2012, ApJL, 747, L26

Sahnow, D. J., et al. 2011, in Society of Photo-Optical Instrumentation Engineers (SPIE) Conference Series, Vol. 8145, Society of Photo-Optical Instrumentation Engineers (SPIE) Conference Series

Savage, B. D., Kim, T.-S., Wakker, B. P., Keeney, B., Shull, J. M., Stocke,

J. T., \& Green, J. C. 2014, ApJS, 212, 8

Savage, B. D. \& Sembach, K. R. 1991, ApJ, 379, 245

Schiminovich, D., et al. 2007, ApJS, 173, 315

Schombert, J. M., McGaugh, S. S., \& Eder, J. A. 2001, AJ, 121, 2420

Shull, J. M., Danforth, C. W., \& Tilton, E. M. 2014, ApJ submitted

Simcoe, R. A., et al. 2011, ApJ, 743, 21

Springel, V. 2005, MNRAS, 364, 1105

Steidel, C. C. 1990 , ApJS, 72, 1

Steidel, C. C., Erb, D. K., Shapley, A. E., Pettini, M., Reddy, N.,

Bogosavljević, M., Rudie, G. C., \& Rakic, O. 2010, ApJ, 717, 289

Stocke, J. T., Keeney, B. A., Danforth, C. W., Shull, J. M., Froning, C. S.,

Green, J. C., Penton, S. V., \& Savage, B. D. 2013, ApJ, 763, 148

Stocke, J. T., et al. 2014, ArXiv e-prints:1405.4307

Thom, C., et al. 2012, ApJL, 758, L41

Tilton, E. M., Danforth, C. W., Shull, J. M., \& Ross, T. L. 2012, ApJ, 759, 112

Tripp, T. M., Aracil, B., Bowen, D. V., \& Jenkins, E. B. 2006, ApJL, 643, L77

Tripp, T. M., Sembach, K. R., Bowen, D. V., Savage, B. D., Jenkins, E. B., Lehner, N., \& Richter, P. 2008, ApJS, 177, 39

Tripp, T. M., et al. 2011, Science, 334, 952

Tumlinson, J. \& Fang, T. 2005, ApJL, 623, L97

Tumlinson, J., Werk, J. K., Thom, C., Meiring, J. D., Prochaska, J. X., Tripp,

T. M., O’Meara, J. M., Okrochkov, M., \& Sembach, K. R. 2011a, ApJ, 733,111

Tumlinson, J., et al. 2011b, Science, 334, 948

Tumlinson, J., et al. 2013, ApJ, 777, 59

Weiner, B. J., et al. 2009, ApJ, 692, 187

Werk, J. K., Prochaska, J. X., Thom, C., Tumlinson, J., Tripp, T. M., O’Meara, J. M., \& Meiring, J. D. 2012, ApJS, 198, 3

Werk, J. K., Prochaska, J. X., Thom, C., Tumlinson, J., Tripp, T. M.,

O’Meara, J. M., \& Peeples, M. S. 2013, ApJS, 204, 17

Werk, J. K., et al. 2014, ApJ, 792, 8

Woo, J., Courteau, S., \& Dekel, A. 2008, MNRAS, 390, 1453

York, D. G., et al. 2000, AJ, 120, 1579

Zhu, G. \& Ménard, B. 2013, ApJ, 770, 130

HST-COS spectra of the detected 17 C IV absorbers over-plotted with their corresponding Voigt profile fits are shown below. 
TABLE 1

COS-DWARfs Galaxy-CIV Measurements:

\begin{tabular}{|c|c|c|c|c|c|c|c|c|c|c|c|c|c|}
\hline QSO Name & Galaxy $^{\mathrm{a}}$ & $\begin{array}{c}\text { Galaxy } \\
\alpha[\mathrm{J} 2000]\end{array}$ & $\begin{array}{c}\text { Galaxy } \\
\delta[\mathrm{J} 2000]\end{array}$ & $z_{\text {sys }}$ & $\mathrm{L} / \mathrm{L} * \mathrm{~b}$ & $\begin{array}{c}\log M_{*} \\
{\left[M_{\odot}\right]}\end{array}$ & $\begin{array}{l}\mathrm{R}^{\mathrm{c}} \\
{[\mathrm{kpc}]}\end{array}$ & $\begin{array}{l}\mathrm{R}_{\mathrm{vir}}{ }^{\mathrm{d}} \\
{[\mathrm{kpc}]}\end{array}$ & $\underset{\left[\mathrm{yr}^{-1}\right]}{\log \mathrm{SSFR}}$ & $\begin{array}{c}\log \left(\mathrm{N}_{\mathrm{CIV}}\right)^{\mathrm{e}} \\
{\left[\mathrm{cm}^{-2}\right]}\end{array}$ & $\begin{array}{c}\mathrm{W}_{\mathrm{r}}^{\mathrm{f}} \\
{[\mathrm{m} \AA]}\end{array}$ & $\begin{array}{c}\phi^{\mathrm{g}} \\
{[\mathrm{Deg}]}\end{array}$ & $\begin{array}{c}\text { C IV } 3 \sigma \\
\text { detection limit }[\mathrm{m} \AA]\end{array}$ \\
\hline $\mathrm{J} 0929+4644$ & 172_157 & 09:29:11.71 & $+46: 41: 47.2$ & 0.017 & 0.019 & 8.5 & 52 & 132 & -8.7 & $13.73 \pm 0.05$ & $173 \pm 22$ & 50 & 30 \\
\hline J0925+4535 & 227_334 & 09:25:30.98 & $+45: 31: 57.8$ & 0.014 & 0.111 & 10.0 & 95 & 259 & -10.3 & $13.56 \pm 0.06$ & $106 \pm 18$ & 9 & 30 \\
\hline $\mathrm{J} 1207+2624$ & 285_98 & $12: 07: 13.89$ & $+26: 24: 55.4$ & 0.048 & 0.066 & 9.7 & 90 & 225 & -10.0 & $14.19 \pm 0.03$ & $437 \pm 33$ & 11 & 36 \\
\hline $\mathrm{J} 1545+0936$ & $285 \_81$ & $15: 45: 48.20$ & $+09: 36: 42.2$ & 0.055 & 0.181 & 9.8 & 84 & 232 & -9.8 & $14.14 \pm 0.05$ & $393 \pm 41$ & 55 & 57 \\
\hline $\mathrm{J} 1342+1844$ & 160_190 & $13: 42: 51.43$ & $+18: 41: 44.5$ & 0.027 & 0.052 & 9.4 & 101 & 202 & -10.0 & $13.71 \pm 0.11$ & $158 \pm 44$ & 65 & 54 \\
\hline $\mathrm{J} 1122+5755$ & 259_309 & 11:22:06.67 & $+57: 54: 45.3$ & 0.011 & 0.029 & 9.1 & 65 & 182 & -10.1 & $13.74 \pm 0.08$ & $149 \pm 39$ & 86 & 51 \\
\hline $\mathrm{J} 1330+3119$ & 70_57 & $13: 30: 57.52$ & $+31: 19: 50.2$ & 0.034 & 0.045 & 9.5 & 37 & 206 & -10.1 & $14.27 \pm 0.03$ & $406 \pm 30$ & 7 & 51 \\
\hline $\mathrm{J} 1134+2555$ & 358_76 & $11: 34: 57.46$ & $+25: 56: 44.6$ & 0.032 & 0.080 & 9.7 & 47 & 224 & -9.9 & $13.58 \pm 0.12$ & $111 \pm 35$ & 78 & 48 \\
\hline $\mathrm{J} 1059+2517$ & 247_92 & $10: 59: 52.49$ & $+25: 16: 33.5$ & 0.021 & 0.117 & 9.9 & 37 & 252 & -10.1 & $13.74 \pm 0.08$ & $152 \pm 34$ & 85 & 42 \\
\hline $\mathrm{J} 0843+4117$ & $94 \_\overline{1} 77$ & $08: 44: 05.20$ & $+41: 17: 26.4$ & 0.030 & 0.040 & 9.6 & 103 & 216 & -9.9 & $13.84 \pm 0.10$ & $180 \pm 43$ & 22 & 75 \\
\hline J0949+3902 & 234_105 & 09:49:45.57 & $+39: 01: 01.9$ & 0.018 & 0.064 & 9.7 & 37 & 233 & -9.7 & $>14.54$ & $605 \pm 28$ & 9 & 42 \\
\hline $\mathrm{J} 0826+0742$ & $69+79$ & $08: 26: 38.51$ & $+07: 43: 16.4$ & 0.052 & 0.085 & 9.7 & 77 & 222 & -9.6 & $>14.39$ & $452 \pm 38$ & 63 & 54 \\
\hline $\mathrm{J} 0959+0503$ & 318_13 & 09:59:15.04 & $+05: 04: 05.0$ & 0.059 & 0.088 & 10.0 & 14 & 247 & -9.9 & $>14.69$ & $824 \pm 38$ & 56 & 42 \\
\hline $\mathrm{J} 1236+2641$ & $356 \_25$ & $12: 36: 03.91$ & $+26: 42: 01.4$ & 0.062 & 0.043 & 9.4 & 28 & 193 & -9.7 & $>14.38$ & $258 \pm 83$ & 50 & 144 \\
\hline J0042-1037 & $358 \_9$ & $00: 42: 22.27$ & $-10: 37: 35.2$ & 0.095 & 0.039 & 9.6 & 15 & 200 & -10.3 & $>14.55$ & $511 \pm 48$ & 27 & 87 \\
\hline PG1202+281 & $165 \_95$ & $12: 04: 43.87$ & $+27: 52: 39.2$ & 0.051 & 0.096 & 10.0 & 92 & 254 & $<-12.1$ & $13.58 \pm 0.10$ & $95 \pm 30$ & 59 & 54 \\
\hline $\mathrm{J} 0820+2334$ & $260 \_17$ & $08: 20: 22.99$ & $+23: 34: 47.4$ & 0.095 & 0.046 & 9.8 & 29 & 218 & $<-10.9$ & $>14.10$ & $201 \pm 42$ & 50 & 87 \\
\hline J0809+4619 & 257_269 & 08:08:42.75 & $+46: 18: 29.0$ & 0.024 & 0.030 & 9.0 & 125 & 169 & -9.6 & $<12.88$ & $<14$ & 52 & 30 \\
\hline $\mathrm{J} 1327+4435$ & $122 \_131$ & $13: 27: 14.52$ & $+44: 33: 54.3$ & 0.048 & 0.089 & 9.8 & 119 & 229 & -9.5 & $<13.25$ & $<19$ & 32 & 45 \\
\hline $\mathrm{J} 0912+2957$ & 20_223 & $09: 12: 41.57$ & $+30: 00: 53.9$ & 0.023 & 0.062 & 9.8 & 102 & 240 & -10.0 & $<12.97$ & $<18$ & 42 & 39 \\
\hline $\mathrm{J} 0947+1005$ & $135 \_580$ & 09:48:00.79 & $+09: 58: 15.4$ & 0.010 & 0.014 & 9.0 & 120 & 172 & -10.0 & $<13.02$ & $<20$ & 73 & 45 \\
\hline PG1049-005 & $316 \_78$ & $10: 51: 47.77$ & $-00: 50: 20.3$ & 0.039 & 0.110 & 9.6 & 58 & 219 & -9.9 & $<12.98$ & $<16$ & 53 & 36 \\
\hline $\mathrm{J} 1521+0337$ & $252-124$ & $15: 21: 31.73$ & $+03: 36: 51.8$ & 0.036 & 0.087 & 9.5 & 87 & 206 & -9.5 & $<13.56$ & $<58$ & 69 & 120 \\
\hline $\mathrm{J} 1451+2709$ & $184 \_526$ & 14:51:05.82 & $+27: 00: 42.3$ & 0.013 & 0.008 & 8.3 & 135 & 114 & -9.8 & $<13.09$ & $<18$ & 81 & 39 \\
\hline $\mathrm{J} 1342+0505$ & 210_241 & $13: 41: 58.41$ & $+05: 01: 55.7$ & 0.025 & 0.038 & 9.4 & 116 & 201 & -9.9 & $<13.35$ & $<17$ & 73 & 39 \\
\hline $\mathrm{J} 1211+3657$ & 312_196 & $12: 11: 02.54$ & $+36: 59: 53.9$ & 0.023 & 0.147 & 10.1 & 90 & 272 & -9.8 & $<13.17$ & $<29$ & 89 & 45 \\
\hline $\mathrm{J} 1121+0325$ & 73_198 & $11: 21: 26.95$ & $+03: 26: 41.6$ & 0.023 & 0.174 & 10.1 & 89 & 277 & -10.2 & $<13.45$ & $<44$ & 7 & 114 \\
\hline $\mathrm{J} 1001+5944$ & 87_608 & 10:02:23.03 & $+59: 44: 34.5$ & 0.011 & 0.018 & 8.7 & 135 & 148 & -9.7 & $<12.89$ & $<15$ & 76 & 30 \\
\hline J0155-0857 & $329 \_403$ & $01: 55: 16.26$ & $-08: 51: 15.4$ & 0.013 & 0.015 & 9.0 & 105 & 169 & -9.8 & $<13.15$ & $<28$ & 24 & 51 \\
\hline J0310-0049 & $124 \_197$ & $03: 10: 38.64$ & $-00: 51: 43.6$ & 0.026 & 0.008 & 8.5 & 101 & 129 & -9.9 & $<13.04$ & $<21$ & 70 & 48 \\
\hline J0242-0759 & 84_223 & 02:43:05.80 & $-07: 58: 53.3$ & 0.029 & 0.040 & 8.9 & 126 & 166 & -9.6 & $<13.23$ & $<34$ & 32 & 72 \\
\hline $\mathrm{J} 1059+1441$ & 31]__200 & 10:59:35.63 & $+14: 44: 07.7$ & 0.010 & 0.006 & 8.2 & 41 & 102 & -9.1 & $<13.06$ & $<16$ & 19 & 36 \\
\hline $\mathrm{J} 1357+1704$ & 93_248 & $13: 57: 29.95$ & $+17: 04: 29.2$ & 0.026 & 0.042 & 9.3 & 124 & 196 & -9.7 & $<12.93$ & $<17$ & 19 & 39 \\
\hline $\mathrm{J} 0946+4711$ & 198_218 & $09: 46: 14.61$ & $+47: 08: 03.3$ & 0.015 & 0.080 & 9.6 & 66 & 222 & -9.9 & $<13.71$ & $<59$ & 18 & 156 \\
\hline $\mathrm{J} 1022+0132$ & $337 \_29$ & $10: 22: 18.22$ & $+01: 32: 45.4$ & 0.074 & 0.024 & 9.1 & 39 & 167 & -10.1 & $<13.31$ & $<45$ & 71 & 72 \\
\hline $\mathrm{J} 1616+4154$ & $327 \_30$ & $16: 16: 47.99$ & $+41: 54: 41.3$ & 0.104 & 0.041 & 9.2 & 55 & 172 & -9.4 & $<13.47$ & $<39$ & 84 & 63 \\
\hline $\mathrm{J} 1356+2515$ & 182_159 & $13: 56: 25.02$ & $+25: 12: 44.2$ & 0.032 & 0.038 & 9.6 & 97 & 212 & $<-12.1$ & $<13.27$ & $<35$ & 87 & 72 \\
\hline $\mathrm{J} 1210+3157$ & 65_308 & $12: 10: 59.71$ & $+31: 59: 12.2$ & 0.022 & 0.022 & 9.3 & 134 & 197 & $<-11.7$ & $<13.11$ & $<25$ & 49 & 57 \\
\hline $\mathrm{J} 1117+2634$ & $114 \_210$ & 11:18:08.60 & $+26: 32: 50.1$ & 0.028 & 0.063 & 9.8 & 114 & 239 & $<-12.1$ & $<13.28$ & $<32$ & 16 & 72 \\
\hline $\mathrm{J} 1104+3141$ & 211_65 & $11: 04: 04.25$ & $+31: 40: 15.1$ & 0.047 & 0.079 & 9.7 & 58 & 225 & $<-10.9$ & $<13.23$ & $<29$ & 16 & 39 \\
\hline $\mathrm{J} 1342+3829$ & $322 \_238$ & $13: 42: 18.70$ & $+38: 32: 12.2$ & 0.012 & 0.031 & 9.2 & 54 & 190 & $<-11.0$ & $<13.07$ & $<19$ & 6 & 42 \\
\hline J0212-0737 & 334_153 & $02: 12: 13.91$ & $-07: 35: 01.1$ & 0.018 & 0.022 & 9.0 & 53 & 170 & $<-10.7$ & $<12.95$ & $<19$ & 21 & 51 \\
\hline $\mathrm{J} 1103+4141$ & 13_58 & $11: 03: 14.15$ & $+41: 42: 51.7$ & 0.030 & 0.029 & 9.5 & 33 & 206 & $<-11.5$ & $<13.34$ & $<25$ & 77 & 45 \\
\hline
\end{tabular}

${ }^{\mathrm{a}}$ We label the galaxies by the position angle with respect to the QSO, N through $\mathrm{E}$, and with the angular separation in arcsec.

${ }^{b}$ Galaxy luminosity in terms of L*, where $\mathrm{L}^{*}$ is given by an R-band absolute magnitude of -21.12 (Blanton et al. 2001).

${ }^{\mathrm{c}}$ Impact parameter in kpc.

$\mathrm{d}$ Virial radius in kpc.

e Limits on $N_{C I V}$ are $2 \sigma$, AOD column Densities

${ }^{\mathrm{f}}$ CIV 1548 rest frame equivalent widths. Limits on $W_{r}$ are $1 \sigma$.

g Azimuthal angle with respect to the galaxy's projected minor axis.

TABLE 2

Minimum CARbon Mass Estimates:

\begin{tabular}{cccc}
\hline \hline Title & $\log M_{*} / \mathrm{M}_{\odot}$ & $\mathrm{M}_{\text {carbon }} / \mathrm{M}_{\odot}{ }^{\mathrm{a}}$ & $\mathrm{M}_{\text {carbon }} / \mathrm{M}_{\odot}{ }^{\mathrm{b}}$ \\
\hline All galaxies & $8-9.5$ & $0.4 \times 10^{6}$ & $0.4 \times 10^{6}$ \\
& $9.5-10$ & $0.9 \times 10^{6}$ & $1.9 \times 10^{6}$ \\
\hline & $8-9.5$ & $0.5 \times 10^{6}$ & $0.5 \times 10^{6}$ \\
Star-forming galaxies & $9.5-10$ & $1.1 \times 10^{6}$ & $2.6 \times 10^{6}$ \\
& & & \\
\hline a AOD $N_{C I V}$ & & \\
b Voigt Profile fitted $N_{C I V}$ & & &
\end{tabular}




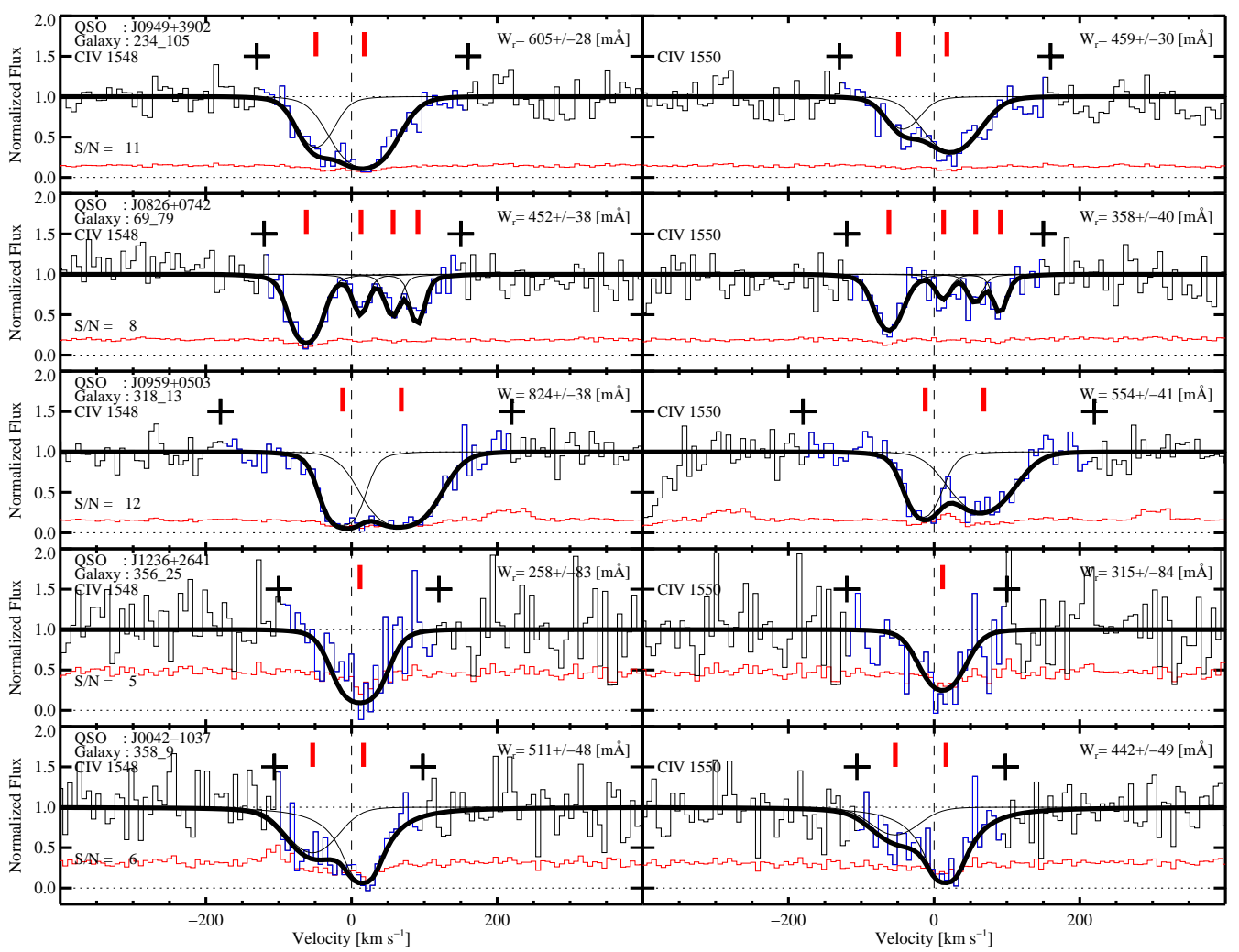

(a)

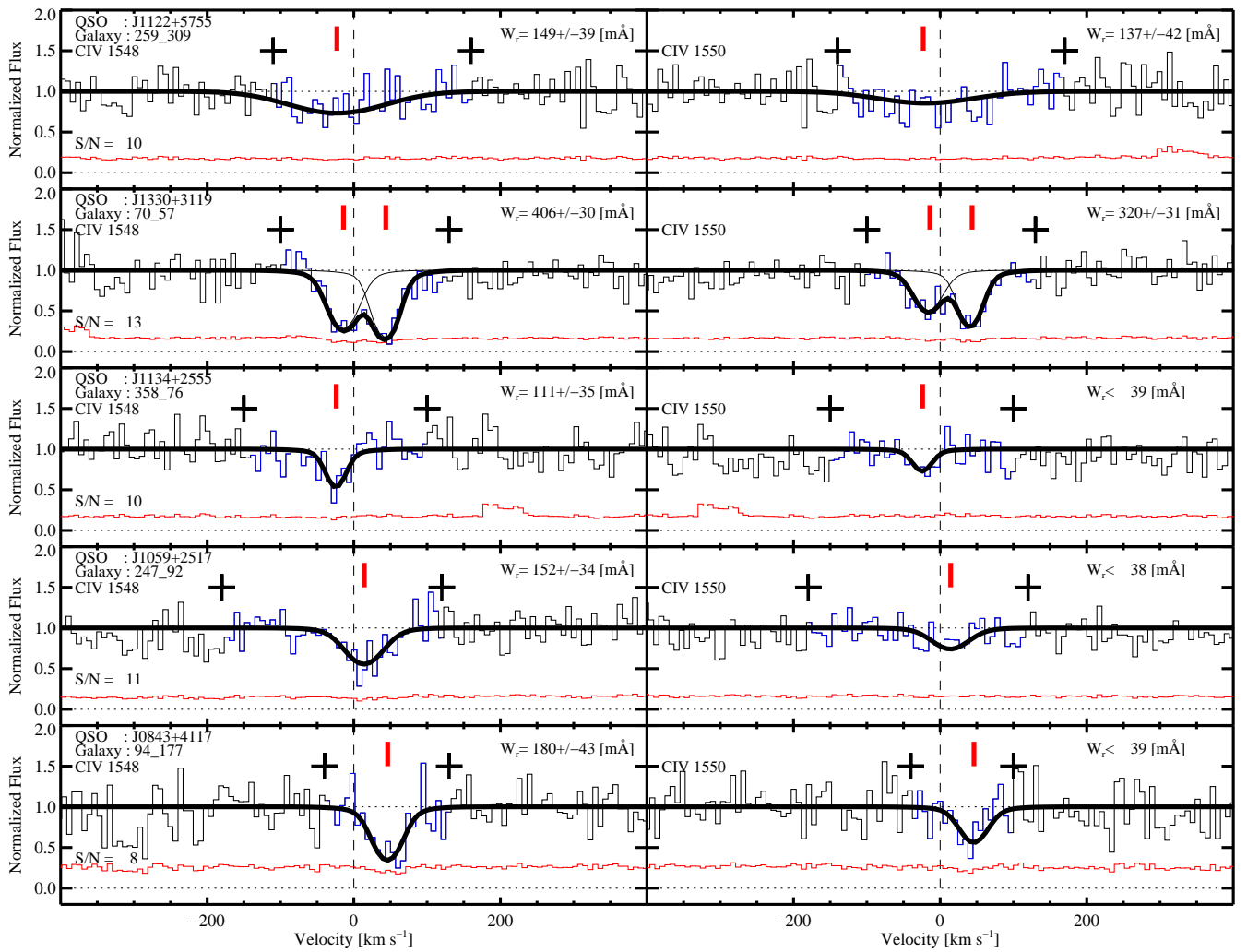

(b)

FIG. 11.- HST-COS quasar spectra of the C IV absorption doublets with their corresponding Voigt profile fits (solid black line). The vertical red ticks indicate the centroids of individual Voigt profile components and the black crosses show the velocity range over which the profile was integrated to compute their equivalent widths. For each system their rest frame equivalent widths and the $\mathrm{S} / \mathrm{N}$ of the spectrum at $\mathrm{C}$ IV rest frame are shown. 


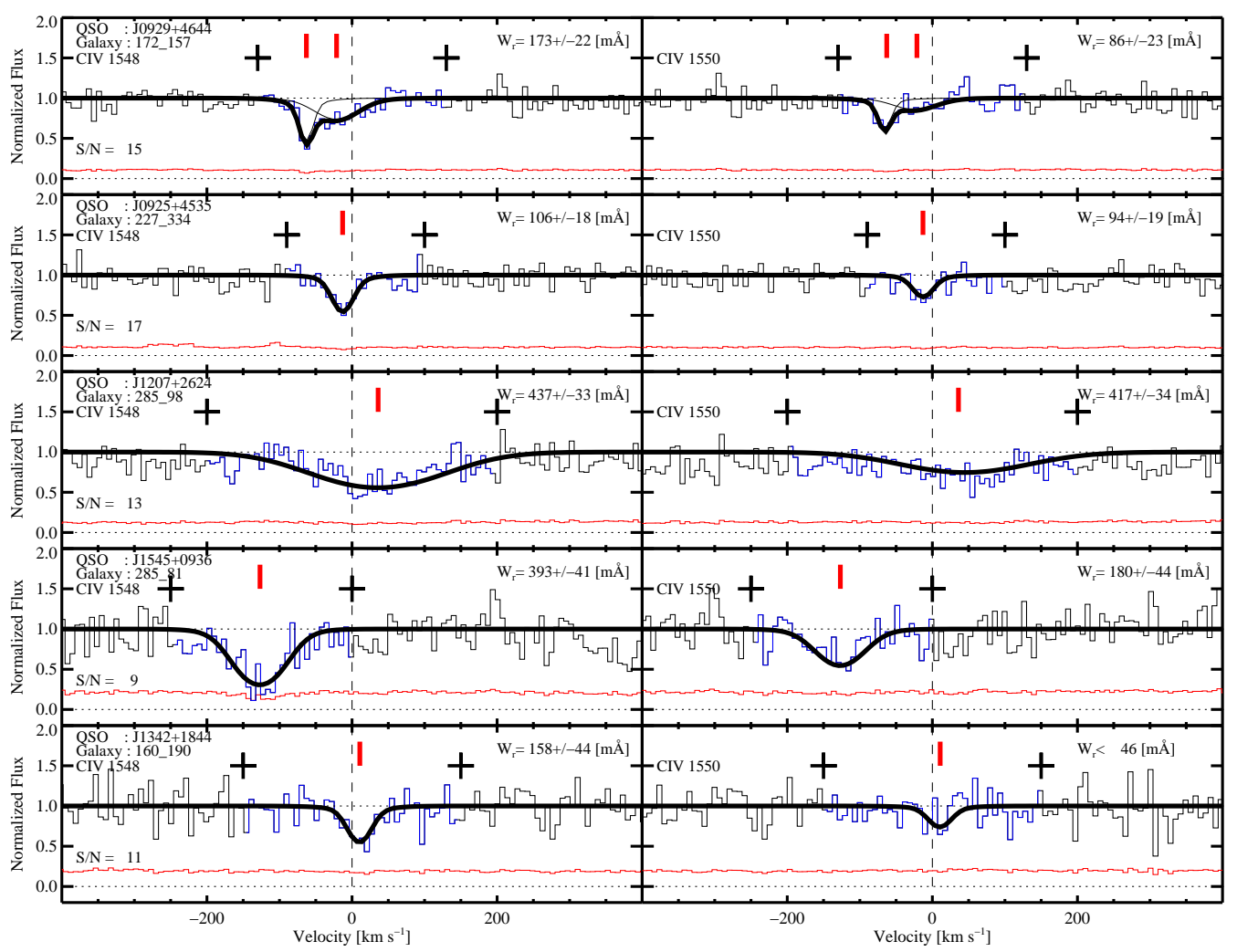

(c)

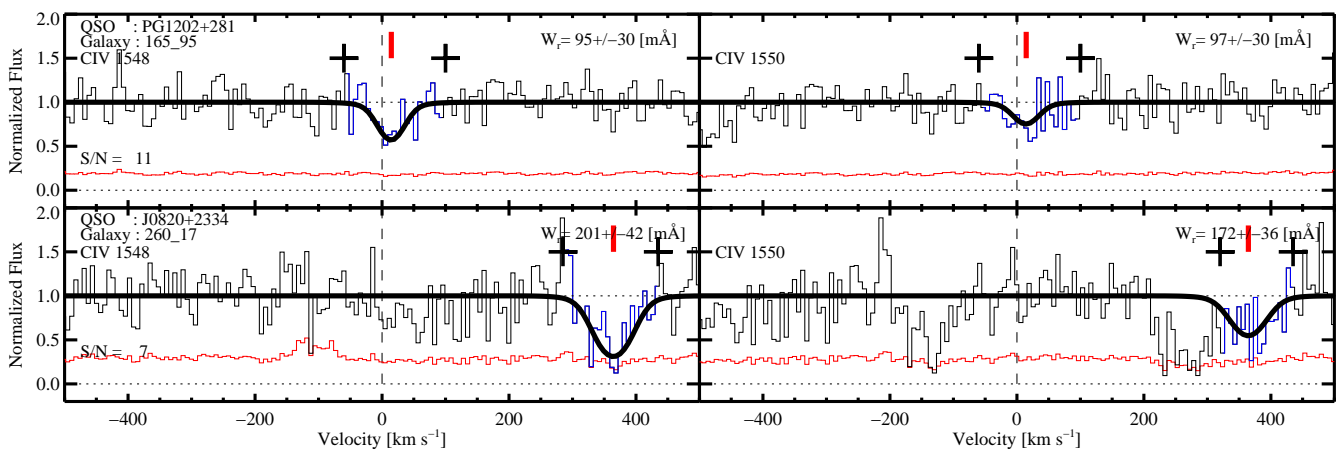

(d)

FIG. 11.- continued 